\title{
LAWA: Loss Aware Workload Assignment in Data Centers
}




\title{
LAWA: LOSS AWARE WORKLOAD ASSIGNMENT IN DATA CENTERS
}

\author{
By Zahra Esmaeilnezhad
}

A Thesis Submitted to the School of Graduate Studies in Partial Fulfilment of the Requirements for the Master of Applied Science

McMaster University (c) Copyright by Zahra Esmaeilnezhad, February 2020 
McMaster University Master of Applied Science (2020) Hamilton, Ontario (Computing and Software)

TITLE: LAWA: Loss Aware Workload Assignment in Data Centers

AUTHOR: Zahra Esmaeilnezhad, M.A.Sc. (McMaster University)

SUPERVISORS: Dr. Douglas G. Down, Dr. Ghada Badawy 


\section{Acknowledgements}

Thanks to Saman Samikermani for all of his love and support. He spent so much time arguing about every point in my work and providing excellent feedback and suggestions. Saman, I am forever grateful for your help.

Thanks to the best supervisor ever, Dr. Douglas. I am truly honoured to be one of his students. Professor Down, you are my role model and the inspiration I needed to grow academically and professionally.

Finally, I need to thank my parents, to whom I owe everything. 


\section{Contents}

\begin{tabular}{llr}
\hline 1 & Introduction & 6
\end{tabular}

\begin{tabular}{lll}
\hline 2 & Literature Review & 11
\end{tabular}

\begin{tabular}{lll}
\hline 3 & Definitions & 17
\end{tabular}

3.1 The Efficiency Function of a Server . . . . . . . . . . . . . . . 17

3.2 The Loss Function of a Single Server . . . . . . . . . . . . . . 20

3.3 Total Load . . . . . . . . . . . . . . . . . . . . . . . . . . 22

3.4 The Total Loss Function . . . . . . . . . . . . . . . . . 23

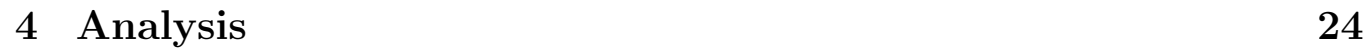

$\begin{array}{lll}5 & \text { Numerical Results } & 62\end{array}$

\begin{tabular}{lll}
\hline 6 & Conclusions & 70
\end{tabular}

\begin{tabular}{lll}
\hline 7 & References & 72
\end{tabular}

\begin{tabular}{|ll}
\hline A Appendix 1 & 76
\end{tabular} 


\section{List of Figures}

1 US data center electricity use [6] $\ldots \ldots \ldots$. . . . . . . . . . . . . . . . 7

$2 \quad$ Estimated energy consumption forecast [2] $\ldots$. . . . . . . . . . 7

$3 \quad$ Projected data center total electricity use [3] . . . . . . . . . . . . 8

$4 \quad$ Total server count in US data centers [3] . . . . . . . . . . . 15

$5 \quad$ HP 750 W CS Platinum Plus power supply [24] . . . . . . . . . 16

$6 \quad$ Analysis of the power consumption of a typical 5000 sq. ft. data center [25] . . . . . . . . . . . . . . . . . . . . 16

$7 \quad$ An example of a downward concave function $\left(f(x)=-x\left(\frac{x-900}{210000}\right)\right.$, $x$ axis is in Watts and $y$ axis is the efficiency) . . . . . . . . . . 19

8 The loss function of the efficiency function corresponding to

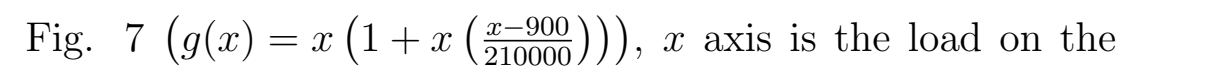
server in Watts and $y$ axis is the loss of the server in Watts)] 21

$9 \quad$ Two of many workload assignments on three servers . . . . . . 25

10 The efficiency curve of HP server model 'D17-310P1A' given in the data sheet . . . . . . . . . . . . . . . . . 63

11 Efficiency at different loads of HP server model 'D17-310P1A' 63

12 Coordinates produced by Engauge Digitizer for HP server model 'D17-310P1A' . . . . . . . . . . . . . . . . 64

13 Curve fitting in Matlab for HP server model 'D17-310P1A' . . 65 


\section{List of Tables}

$1 \quad$ Notation . . . . . . . . . . . . . . . . . . . . . 18

$2 \quad$ Comparing losses for HP server model D17-310P1A . . . . . 67

$3 \quad$ Comparing losses for Dell server model PS-2112-2L $\ldots \ldots$. . . 68

$4 \quad$ Comparing losses for Dell server model D750E-S7 . . . . . . . 69

5 Comparing losses for Dell server model C1100P-00 . . . . . . . 69

$6 \quad$ Comparing losses for Dell server model L600E-S0 . . . . . . . 69

7 Comparing losses for Dell server model DPSN-300 DB C $\ldots 70$ 


\section{Introduction}

Data centers are the backbone of computing, storing, processing, distributing and allowing access to large amounts of data [1]. As of 2018, each year data centers consume approximately 200 TWh of energy, more than the national energy consumption of countries such as Iran [2]. U.S. data centers consumed about 70 TWh in 2014, 1.8 percent of U.S. electricity consumption [3]. Fig. 1 shows the amount of energy used in all U.S. data centers [6]. Fig. 2 shows estimated energy consumption of information and communications technology up to 2030, where we can see that an increasing proportion of energy consumption is predicted to be due to data centers.

Beyond the direct energy costs to data center owners, this large amount of energy consumption causes air pollution, so reducing power consumption is a "win-win" situation. The end result is a reduction of the impact on the climate and reduction of costs for data center operators [4, 5]. A lot of work has been done to increase the energy efficiency of data centers. Without these energy efficiency improvements, both the consumed and predicted power consumption would significantly increase (Fig.3). More than 600 TWh have been saved over the past two decades because of these energy efficiency improvements [3].

Research on reducing the power consumption of data centers has examined 


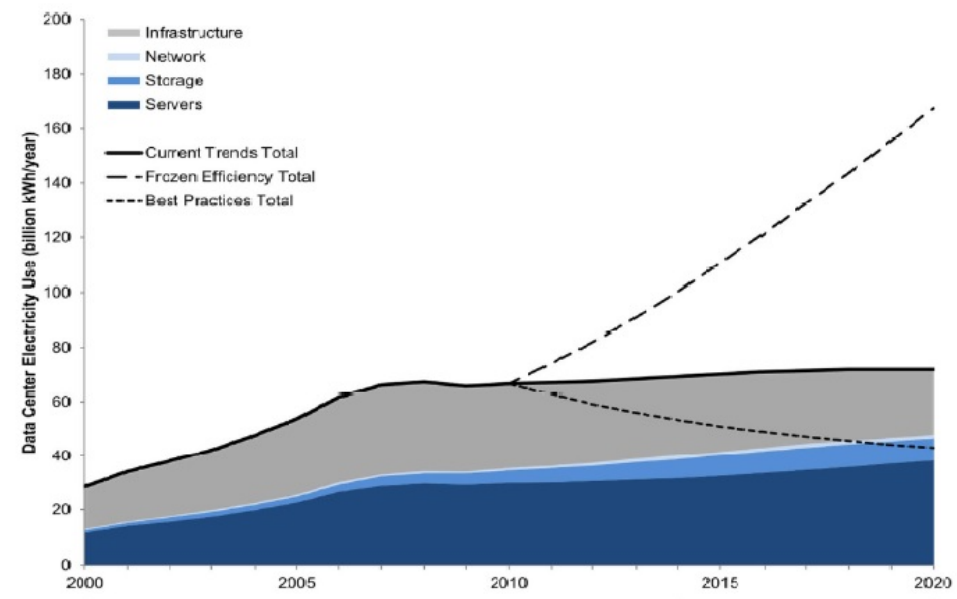

Figure 1: US data center electricity use [6]

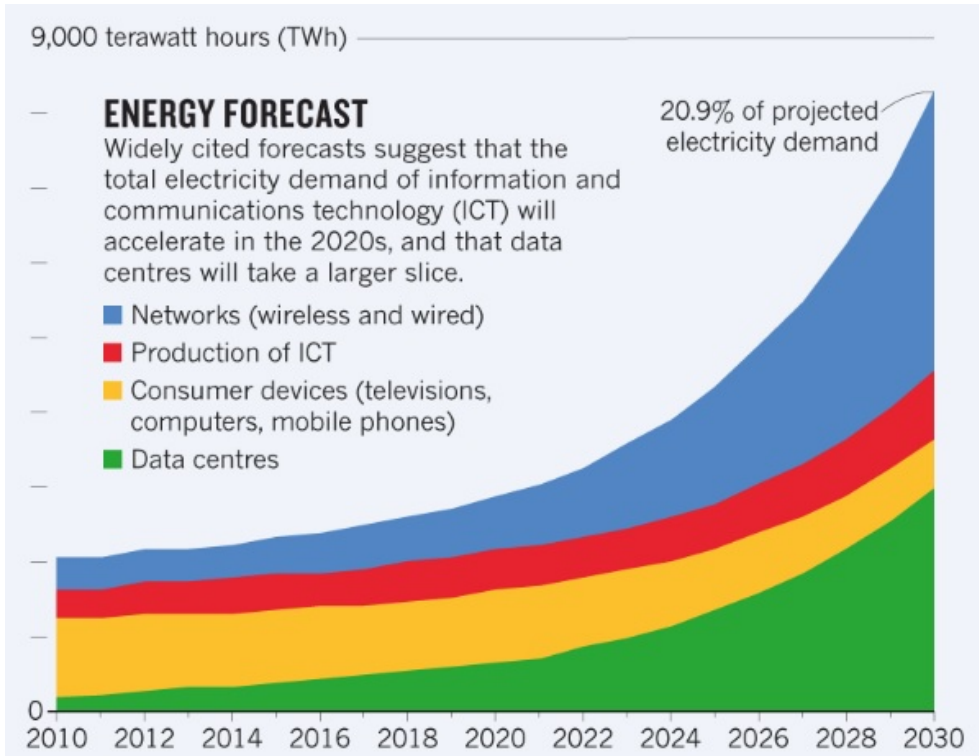

Figure 2: Estimated energy consumption forecast [2] 


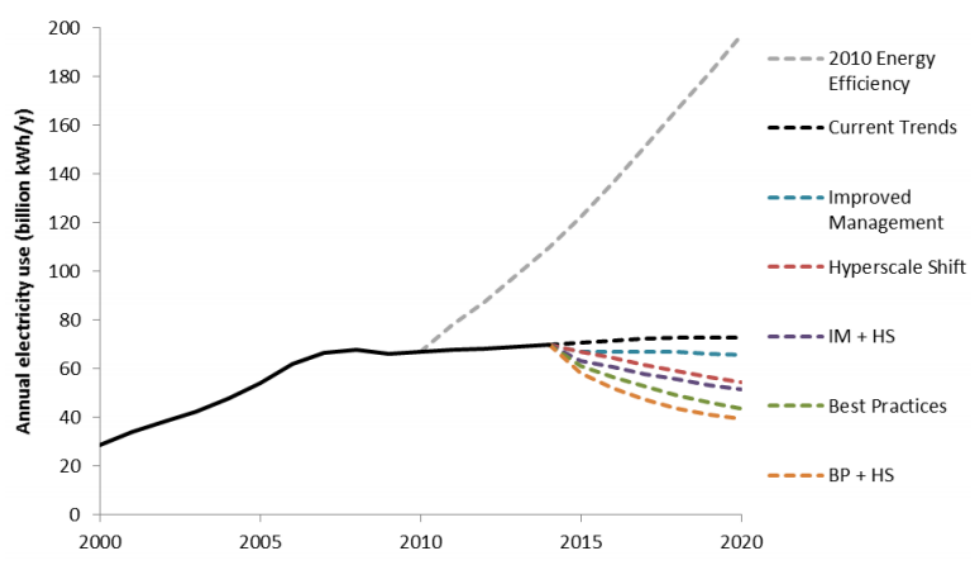

Figure 3: Projected data center total electricity use [3]

different levels of operations. Some proposes algorithms in lower levels such as at the device level and some in higher levels such as servers, cooling systems and workload managers. But as far as we know, there is no work that has considered inefficiencies in power distribution to servers (server power loss). We are looking for algorithms that minimize server power loss and then we would like to compare the resulting algorithms to those that do not consider server power loss to determine whether the resulting algorithms are consistent or not. Note that from now on, we use "loss" instead of "power loss".

The reason that we focus on server losses is that in terms of efficiency, the other components in the power distribution system in a data center both use less power and tend to be much more efficient. We considered the loss of different components of a data center such as servers, uninterruptible power supplies (UPS) and power distribution units (PDU). IT equipment (servers) 
consumes about 52 percent of the total energy consumption of data centers, compared to just one percent for PDUs. Fig. 6 shows the power consumption breakdown in a data center.

The loss of a server is not constant. It is a function of the load on the server (as will be seen in Chapter 3) which means that the workload assignment algorithm can have a significant impact on overall server loss. The dependence on the load means that the problem of workload assignment to minimize server loss is a nontrivial one. We will see that our proposed solution that while simple, solves a problem that at first glance appears to involve more of a subtle trade-off. Also there is a lot of work on workload assignment and given that none of it takes power losses into account, one needs to know whether the effect of power losses is either synergistic (even more is saved than claimed) or antagonistic (less is saved than claimed).

Our goal in this work is to devise a new energy-aware workload assignment algorithm to minimize the server power loss in data centers. We will see that the power savings of such an algorithm can be quite significant and as a result we believe that it is crucial to include such considerations when performing workload assignment. Looking at Fig. 4 the total number of servers is increasing dramatically over the years. So while looking at one (or a small number) of servers, the savings presented here may be thought of as insignificant, when one scales this up to the numbers of servers in Fig. 4, the aggregated savings are significant. 
The amount of power that we are saving using LAWA is significant particularly when applied to older servers, where the efficiency has significant variation with respect to the load (the efficiency curves of older servers look like the blue curve in Fig. 5p, so our approach is particularly effective for large data centers that contain older servers. 


\section{Literature Review}

Here we will give a brief overview of some of the previous work to reduce the power consumption of data centers. This is necessarily not comprehensive, but does provide an idea of the scope of research in this domain.

At the low level, examples of approaches for power savings include different SRAM configurations [7] or using low power modes in network devices, for example in periods of low activity using lower power modes (sleep mode) in some components on LAN switches [8, 9].

In [14] $\mathrm{Xu}$ et al. find that cache power consumption could be slashed by a factor of 25 through changes to caches that are storing commonly used queries in data centers. This is a significant reduction, as in [13] it has been estimated that caches consume 10 percent of the energy resources in a data center.

A significant amount of work has been done at higher levels, the most relevant for this thesis is workload management. In all of the proposed workload management algorithms, the key elements to be determined are the number of servers that need to be turned on and the algorithm to assign the load to these servers to reduce the power consumption of some part of a data center. Here we are going to briefly mention some of the previous works in this area. 
Mirhoseini.Nejad et al. [10] address the reduction of wasted power in data centers due to inefficiencies in workload management, in particular the lack of coordination between IT equipment and cooling units and heterogeneities within the system. They propose a workload assignment algorithm that assigns the workload to those servers that have lower cooling and power requirements. Their proposed algorithm reduces the total power consumption, in particular compared to uniform workload distribution.

Liang et al. [11] also propose a thermal-aware workload distribution policy to reduce the power consumption of clusters in data centers without reducing the quality of service (QoS). Their idea is to give rankings to nodes (hot spots) according to the layout of CRAC units (central air conditioning) and based on these rankings, the load is distributed. To avoid overheating some nodes, after a fixed time interval, the ordering of the nodes will change according to the current monitored temperature.

Another thermal-aware task scheduling algorithm to reduce power costs is described in Tang et al. [16]. In this paper, the thermal environment of a data center is an important factor in determining energy consumption. They suggest that assigning the workload to the coolest locations may not be a good idea because it does not take heat recirculation into account. The hot air that is produced by servers can recirculate from the outlets to the inlets of servers and create hot spots that will increase the energy required by the cooling equipment. As a result, the authors propose a thermal-aware 
task scheduling algorithm to yield an even inlet temperature distribution for servers and as a result considerably reduce the power consumption of cooling units.

Arroba et al. 12 introduce a frequency-aware consolidation algorithm to reduce power consumption. They believe that Dynamic Voltage and Frequency Scaling (DVFS) and consolidation are the two key strategies for energy efficiency in the face of workload variations. They demonstrate that by effectively using these mechanisms, the power consumption of data centers can be reduced.

The idea of consolidating the load on servers by turning them ON and OFF is a common idea. Many researchers have worked on eliminating the idle power consumption of servers by transitioning between a high performance active-state and a very low power state, see for example, [15].

Similar work is described in Pakbaznia and Pedram [17]. They address minimizing the power cost by considering both cooling and IT power usage. As part of their workload assignment policy, they turn various servers ON and $\mathrm{OFF}$ and set the voltage-frequency level for each of the servers. The result is reduced server power consumption and a consequence is that the cooling power consumption is reduced by setting a higher supplied cold air temperature value. 
Additional work in this direction has been performed in [18] and [19], where the authors discuss reducing power consumption through server consolidation, dynamically choosing the number of servers required and turning off the unneeded ones during periods of low load.

In contrast to server consolidation, Meisner et al. 20] suggest assigning the load equally between servers. They demonstrate that transitions between full-system high-performance active-state and low-power inactive-state are inappropriate for Online Data-Intensive (OLDI) services - OLDI is a class of workloads that is very latency sensitive and represents a large volume of data in most data centers. An example of OLDI workloads is advertising. As OLDI workloads are very latency sensitive, turning off the servers is infeasible and ineffective in these settings. They suggest that by balancing the workload on the system, energy-proportionality with an acceptable query latency can be achieved.

The two workload assignment approaches discussed so far (server consolidation and uniform workload distribution) are common nowadays. Some data center operators are willing to use uniform workload distribution due to reasons like minimizing the response time for users and avoiding overload of servers [21]. On the other hand, some data center operators prefer consolidating the load on the minimum number of servers to save costs on maintenance and operation such as physical support, electricity usage, real estate and taxes [22]. 


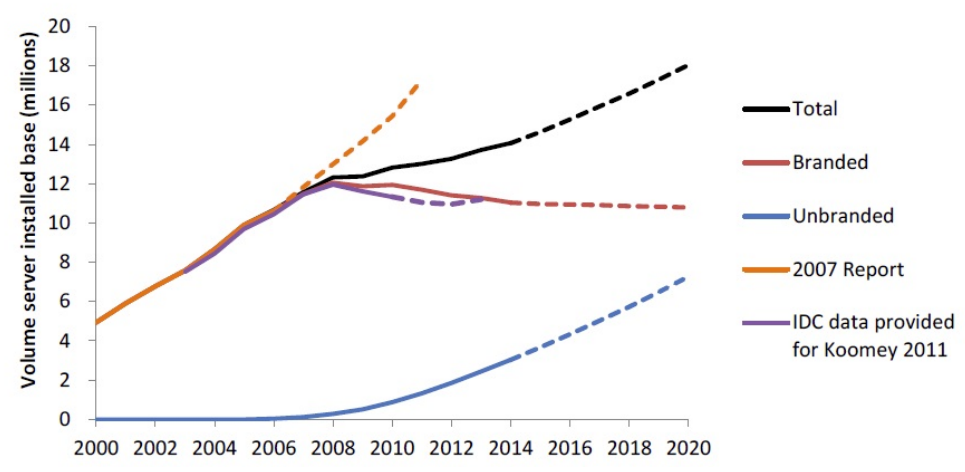

Figure 4: Total server count in US data centers [3]

Our algorithm, Loss Aware Workload Assignment (LAWA), is something in between server consolidation and uniform workload distribution. As in the described work in workload management, we find the number of needed servers and assign the load to these servers to minimize the total loss over all servers, resulting in a significant decrease in power consumption, more than the amount saved in the two approaches described above, particularly server consolidation.

The remainder of this thesis is organized as follows. In Chapter 3, we define some important variables and functions. In Chapter 4 we provide our algorithm and provide a proof of its optimality. Chapter 5 compares LAWA with uniform load distribution and server consolidation, suggesting where LAWA is or is not consistent and the magnitude of power that we are saving when LAWA is not consistent with these approaches. 


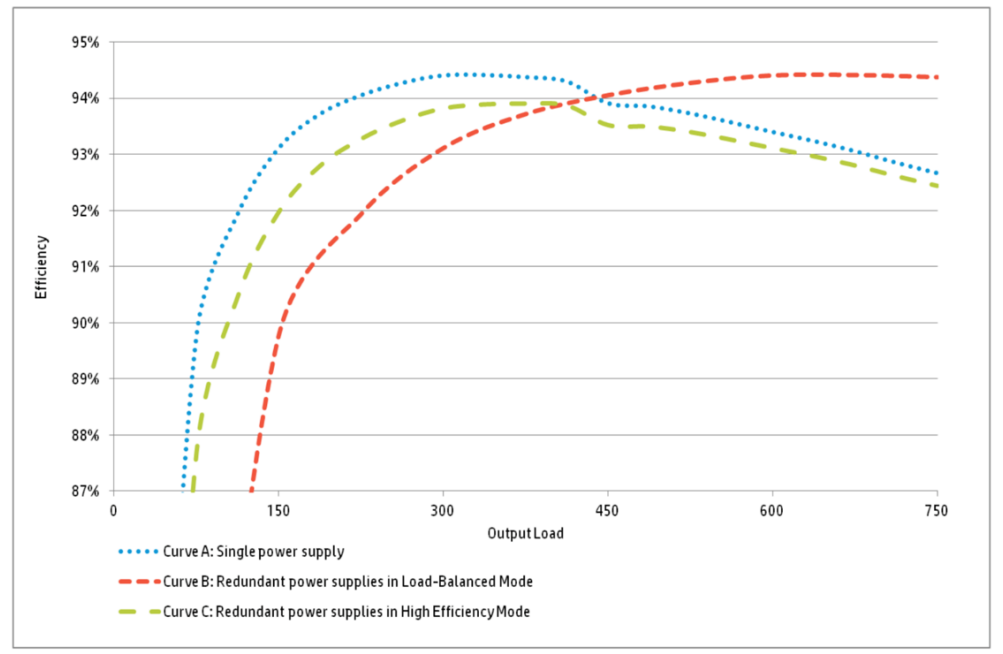

Figure 5: HP 750W CS Platinum Plus power supply [24]
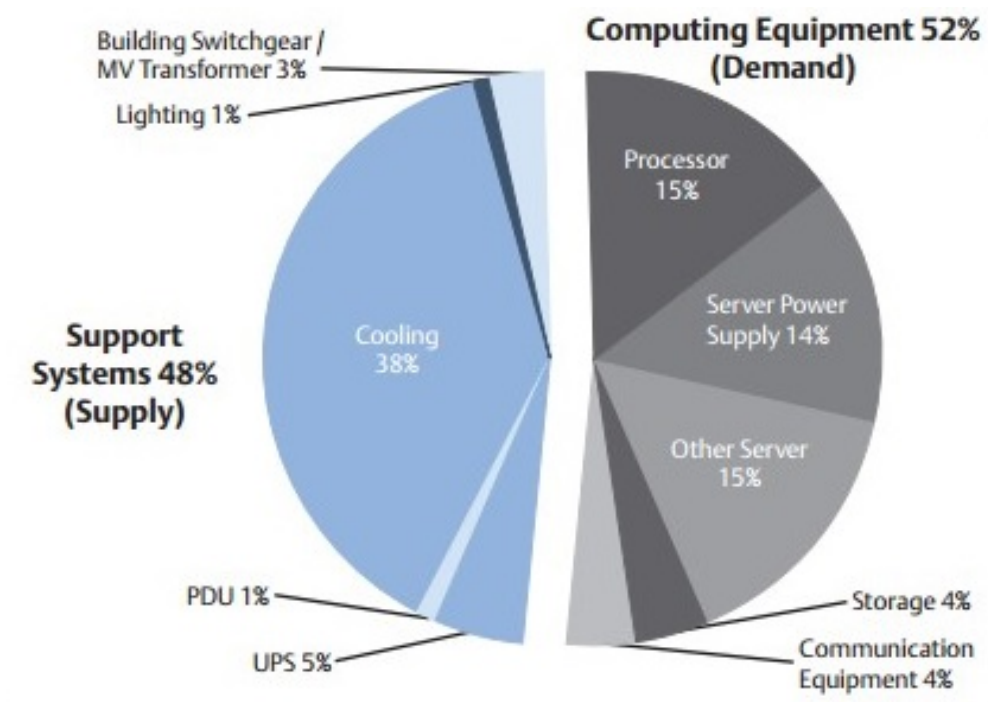

Figure 6: Analysis of the power consumption of a typical 5000 sq. ft. data center $[25]$ 


\section{Definitions}

Before providing our algorithm and the proof of its optimality, it is useful to define some functions and variables that we are going to use frequently.

Recall that we are trying to minimize the total loss over all servers in a data center. In this section we will discuss the form that efficiency functions have and based on the efficiency function, we can find a server's loss function and analyse its behavior. We will also define the total loss function (measured over all servers) and some additional variables that we are going to use later. Table 1 summarizes the variables that we are going to use.

\subsection{The Efficiency Function of a Server}

We looked at hundreds of different servers from different manufacturers [23]. Without exception, the efficiency is a concave function which reaches a maximum at a point that we will define as $x_{\max }$. We will call the efficiency function $f(x)$. The precise shape of $f(x)$ differs from server to server, newer servers tend to have flatter curves compared to older ones. To have a general algorithm which covers all cases, we considered servers whose efficiencies follow the general concave form that is first increasing then decreasing, such as the curve shown in Fig. 7 . 
Table 1: Notation

\begin{tabular}{|c|c|}
\hline Variable & Definition \\
\hline$N$ & Number of available servers \\
\hline$n$ & Number of active (turned on) servers which have load assigned to them $(n \leq N)$ \\
\hline$L$ & Amount of load to be assigned to servers (Watts) \\
\hline$V$ & Maximum capacity of a server (Watts) \\
\hline$x$ or $x_{i}$ & Amount of load on a single server (Watts) \\
\hline$f(x)$ & Efficiency function of a single server \\
\hline$x_{m a x}$ & Amount of load on a single server which maximizes $f(x)$ (Watts) \\
\hline$g(x)$ & Loss function of a single server (Watts) \\
\hline$m$ & Inflection point of $g(x)$ \\
\hline$G(\bar{x})$ & Total loss over all servers (Watts) \\
\hline$K$ & $\frac{L}{x_{\max }}$ \\
\hline$K_{1}$ & $\left\lfloor\frac{L}{x_{\max }}\right\rfloor$ \\
\hline$K_{2}$ & $\left\lceil\frac{L}{x_{\max }}\right\rceil$ \\
\hline$U$ & Utilization of the system \\
\hline
\end{tabular}




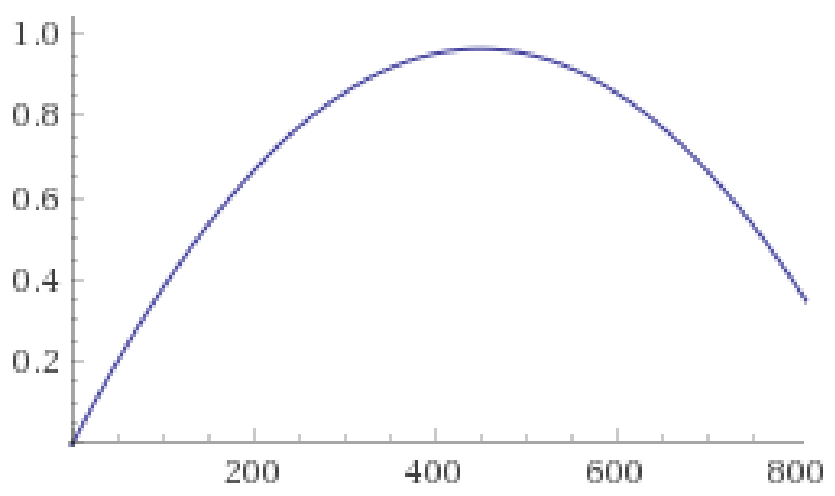

Figure 7: An example of a downward concave function $\left(f(x)=-x\left(\frac{x-900}{210000}\right)\right.$, $x$ axis is in Watts and $y$ axis is the efficiency)

We choose the general form of the function $f(x)$ to be ( $V$ is the maximum capacity of the server):

$$
f(x)=\tilde{A} x^{2}+\tilde{B} x+\tilde{C}, \quad 0<x \leq V .
$$

We first show that $\tilde{A}$ and $\tilde{B}$ must be in a certain range to yield a valid efficiency function.

Lemma 1. For a valid efficiency function, $\tilde{A}<0$ and $\tilde{B}>0$.

\section{Proof.}


To find the signs of $\tilde{A}$ and $\tilde{B}$ we argue based on the signs of $x_{\max }$ and the second derivative of $f(x)$. As the efficiency function is concave, its second derivative is negative $\left(f^{\prime \prime}(x)<0\right)$ so let us find the second derivative of $f(x)$ :

$$
f^{\prime}(x)=2 \tilde{A} x+\tilde{B} \Rightarrow f^{\prime \prime}(x)=2 \tilde{A} .
$$

Since $f^{\prime \prime}(x)<0$ we can find the sign of $\tilde{A}$ :

$$
2 \tilde{A}<0 \Rightarrow \tilde{A}<0
$$

To find the sign of $\tilde{B}$, we can use the fact that $x_{\max }>0$. We know $x_{\max }$ is always positive because $x_{\max }$ is the amount of load on the server that maximizes the efficiency. Let us calculate the value of $x_{\max }$ :

$$
f^{\prime}(x)=0 \Rightarrow 2 \tilde{A} x+\tilde{B}=0 \Rightarrow x_{\max }=-\frac{\tilde{B}}{2 \tilde{A}}
$$

Thus, $-\frac{\tilde{B}}{2 \tilde{A}}>0$, and as we have previously shown that $\tilde{A}<0$, then $\tilde{B}>0$.

\subsection{The Loss Function of a Single Server}

Now that we have the formula of the efficiency function, (see (1)) for a single server, we can calculate the loss function of a single server. If $g(x)$ is the loss 


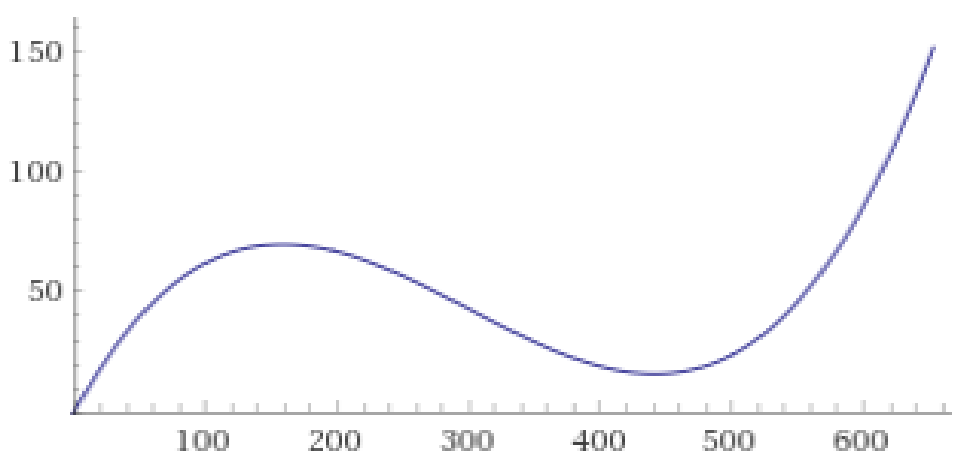

Figure 8: The loss function of the efficiency function corresponding to Fig. $7\left(g(x)=x\left(1+x\left(\frac{x-900}{210000}\right)\right)\right), x$ axis is the load on the server in Watts and $y$ axis is the loss of the server in Watts)

function of a server in Watts then $g(x)$ is:

$$
\begin{aligned}
g(x) & =x(1-f(x)) \\
& =x\left(1-\left(\tilde{A} x^{2}+\tilde{B} x+\tilde{C}\right)\right) \\
& =-\tilde{A} x^{3}-\tilde{B} x^{2}+x(1-\tilde{C}) .
\end{aligned}
$$

If we define $A=-\tilde{A}, B=-\tilde{B}$ and $C=1-\tilde{C}$ then the general form of $g(x)$ is:

$$
g(x)=A x^{3}+B x^{2}+C x, A>0, B<0,0<x \leq V .
$$

An example of $g(x)$ is shown in Fig. 8. Note that we can also rewrite $x_{\max }$ in (2): 


$$
x_{\max }=-\frac{B}{2 A} .
$$

One important variable that we are going to use is the inflection point of $g(x)$. Let us call the inflection point $m$, the point which makes the second derivative of $g(x)$ zero:

$$
g^{\prime \prime}(x)=6 A x+2 B=0 \Rightarrow m=-\frac{B}{3 A} .
$$

\subsection{Total Load}

$L$ is the amount of load (in Watts) that we are going to assign to servers. It will be convenient to define $L=\alpha m$, where $\alpha$ is a positive real number. By substituting for $m$ using (5):

$$
L=-\frac{B \alpha}{3 A} .
$$




\subsection{The Total Loss Function}

Now that we have the loss function of a single server, the total loss function is the sum of the individual servers' loss functions. We assume that all of the servers are the same so they have the same efficiency (and hence loss) function. If $\bar{x}=\left[x_{1}, x_{2}, \cdots, x_{n}\right]$, then the total loss is thus defined as

$$
G(\bar{x})=g\left(x_{1}\right)+g\left(x_{2}\right)+\cdots+g\left(x_{n}\right), 0<x_{1}, \cdots, x_{n} \leq V .
$$

Since the total load that is assigned to servers is $L$ then $x_{1}+x_{2}+\cdots+$ $x_{n-1}+x_{n}=L$, so $x_{n}$ can be determined given $x_{1}$ through $x_{n-1}$, i.e., $G(\bar{x})$ is a function of $n-1$ variables:

$$
\begin{aligned}
G(\bar{x})= & g\left(x_{1}\right)+g\left(x_{2}\right)+\cdots+g\left(x_{n-1}\right)+g\left(L-x_{1}-x_{2}-\cdots-x_{n-1}\right), \\
& 0<x_{1}, \cdots, L-x_{1}-x_{2}-\cdots-x_{n-1} \leq V .
\end{aligned}
$$




\section{Analysis}

Now that we have defined the variables and functions that we require, we are going to introduce a workload assignment algorithm which minimizes $G(\bar{x})$. Our proposed algorithm assigns $x_{\max }$ (or as close to $x_{\max }$ as possible) or 0 (if there are $n$ active servers, then $N-n$ servers are turned off (have load 0$)$ ) to each server. At first, it might seem obvious that this is the optimal workload assignment, but it is not if we look at $g(x)$. For example, suppose that we are going to assign $L$ to three servers, two possible configurations are shown in Fig. 9. Each point is the load on a server and there are two different configurations (red and green). By visual inspection, it is not obvious which configuration results in a smaller value of $G(\bar{x})$. However, we will see below (after some work), that the intuitive solution is optimal. In particular, we propose the following Loss Aware Workload Assignment (LAWA) algorithm. Let $K_{1}=\left\lfloor\frac{L}{x_{\max }}\right\rfloor$ and $K_{2}=\left\lceil\frac{L}{x_{\max }}\right\rceil$ :

- If $K_{1} \leq N$ and $K_{2} \leq N$ then assign $L$ equally to either $K_{1}$ or $K_{2}$ servers (the value with the lower loss).

- If $K_{1}=N$ and $K_{2}>N$ then assign $L$ equally to $K_{1}$ servers.

- If $K_{1}>N$, assign $L$ equally to $N$ servers. 


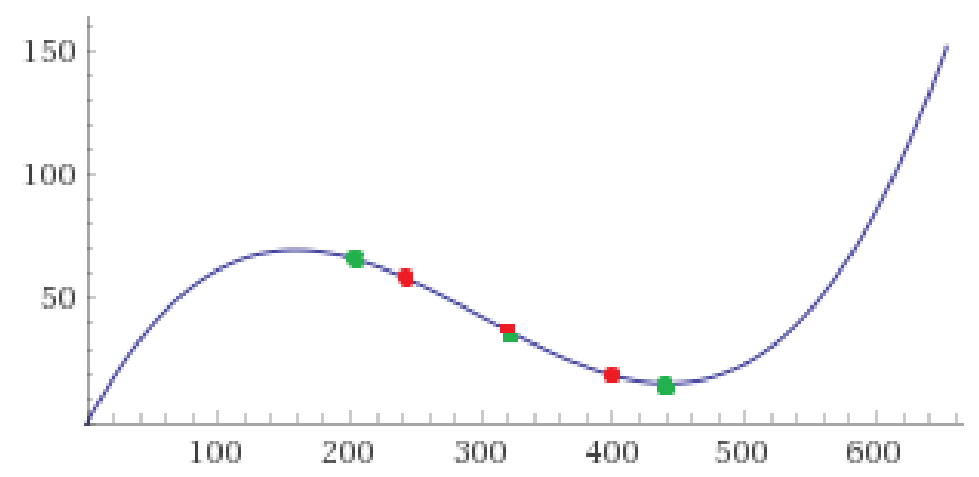

Figure 9: Two of many workload assignments on three servers

The first case in LAWA is a form of server consolidation, but not as extreme as the case when one is trying to consolidate the load on the minimum number of servers and the last two cases coincide with uniform load balancing. Theorem 1 shows for sufficiently large $L$, LAWA is minimizing $G(\bar{x})$.

Theorem 1. For sufficiently large L, LAWA is optimal.

In order to prove Theorem 1, we have to perform a number of intermediate steps. To minimize $G(\bar{x})$, we have to find the solutions of (8) and we also need to check the end points of $G(\bar{x})$. In Lemma 2, we will show that the minimum of $G(\bar{x})$ is not at any of the end points. In Lemma 3, we find the solutions of (8) and in Lemma 5 and Lemma 6 we will show that the minimum is following the format of the solutions provided in Lemma 3. Then in Lemma 
7 and Lemma 8, we explicitly identify the minimum. Note that the condition on $L$ in Theorem 1 is discussed in more detail at the end of Lemma 6. So let us start with checking end points. The end points of $G(\bar{x})$ are $x_{i}=0$ and $x_{i}=V$. We supposed servers with no load are turned off $\left(x_{i}>0\right.$, see (7)) so we are not concerned about the end point $x_{i}=0$. The following lemma addresses the case that some servers have load $V$. Note that in the following lemma, we suppose that $x_{\max }<\frac{3}{4} V$ and this is a reasonable assumption because usually $x_{\max } \leq \frac{V}{2}$.

Lemma 2. Suppose $x_{\max }<\frac{3}{4} V$. Any $\bar{x}$ which minimizes $G(\bar{x})$, must have all entries $x_{i}<V$.

\section{Proof.}

Suppose that we have two servers where one of them is full $(x=V)$ and the other one has load $W<V$. We are going to show that if we decrease the load on the first server by $\epsilon$ and add it to the second server, the loss of the second configuration would be better (i.e. $g(V)+g(W)>g(V-\epsilon)+g(W+\epsilon)$ ) so having a server at load $x_{i}=V$ cannot minimize $G(\bar{x})$. Recall that $g(x)$ is a cubic function (3) so before truncating the domain of $g(x)$ from zero to $V$, $g(x)$ is symmetric with respect to the inflection point $m$. Also since $g(x)$ is a cubic function and $A>0(3), g^{\prime}(x)$ is an upward quadratic function and its minimum (the point that makes the derivative of $g^{\prime}(x)$ equal to zero (i.e $\left.\left.g^{\prime \prime}(x)=0\right)\right)$ is $m$. So $g^{\prime}(x)$ is increasing by moving to the right of the curve from $m$ and by moving to the left from $m$. If we truncate $g^{\prime}(x)$ from $x=0$ 
to $x=V$, at the end of the interval (which is $V$ ), $g^{\prime}(V)$ is the maximum in the interval $[m, V]$. Since $x_{\max }<\frac{3}{4} V$ then using (4) and (5), $m<\frac{V}{2}$, which means that in the interval of $[0, V], V$ is the furthest point from $m$ so $g^{\prime}(V)$ is greater than $g^{\prime}(x)$ for all other points of the interval $[0, V)$. So if we move to the left by $\epsilon$ from $V, g(x)$ will drop more than moving to the right from any point before $V$ by $\epsilon$, so $g(V)-g(V-\epsilon)>g(W+\epsilon)-g(W)$. So there exists a better load assignment than filling a server.

Now that we proved that the end points cannot determine the minimum of $G(\bar{x})$, let us start finding the solutions of $(8)$ to find the minimum of $G(\bar{x})$.

$$
\frac{\partial G(\bar{x})}{\partial x_{\ell}}=0 \quad \ell=1,2, \cdots, n-1 .
$$

The solutions of (8) are characterized in the following lemma. Note that in the following lemmas we suppose that $n \geq 3$, since the number of servers in data centers is large, this assumption is reasonable. In Appendix 1, we will examine the case $n=2$.

Lemma 3. For each $\ell$, (8) has the following two solutions:

$$
x_{\ell}=\frac{L-\sum_{j=1, j \neq \ell}^{n-1} x_{j}}{2}
$$




$$
\sum_{j=1, j \neq \ell}^{n-1} x_{j}=L-2 m .
$$

\section{Proof.}

From (7), for each $x_{\ell}$ two terms of $G(\bar{x})$ are dependent on $x_{\ell}$, one is $g\left(x_{\ell}\right)$ and the other is $g\left(L-x_{1}-x_{2}-\cdots-x_{\ell}-\cdots-x_{n-1}\right)$ so:

$$
\frac{\partial G(\bar{x})}{\partial x_{\ell}}=\frac{\partial g\left(x_{\ell}\right)}{\partial x_{\ell}}+\frac{\partial g\left(L-x_{1}-x_{2}-\cdots-x_{\ell}-\cdots-x_{n-1}\right)}{\partial x_{\ell}}
$$

Without loss of generality, we will determine the solution for $\frac{\partial G(\bar{x})}{\partial x_{1}}$. We calculate $\frac{\partial g\left(x_{1}\right)}{\partial x_{1}}$ and $\frac{\partial g\left(L-x_{1}-x_{2}-x_{3}-\cdots-x_{n-1}\right)}{\partial x_{1}}$ separately by using (3), then adding them together.

Finding $\frac{\partial g\left(x_{1}\right)}{\partial x_{1}}$ :

$$
\frac{\partial g\left(x_{1}\right)}{\partial x_{1}}=3 A x_{1}^{2}+2 B x_{1}+C
$$

Similarly, for $\frac{\partial g\left(L-x_{1}-x_{2}-x_{3}-\cdots-x_{n-1}\right)}{\partial x_{1}}$ : 


$$
\begin{aligned}
& \frac{\partial g\left(L-x_{1}-x_{2}-x_{3}-\cdots-x_{n-1}\right)}{\partial x_{1}} \\
& \quad=-3 A\left(L-x_{1}-x_{2}-x_{3}-\cdots-x_{n-1}\right)\left(L-x_{1}-x_{2}-x_{3}-\cdots-x_{n-1}\right) \\
& \quad-2 B\left(L-x_{1}-x_{2}-x_{3}-\cdots-x_{n-1}\right)-C
\end{aligned}
$$

Expanding $\left(L-x_{1}-x_{2}-x_{3}-\cdots-x_{n-1}\right)\left(L-x_{1}-x_{2}-x_{3}-\cdots-x_{n-1}\right)$ and $-2 B\left(L-x_{1}-x_{2}-x_{3}-\cdots-x_{n-1}\right)$ :

$$
\begin{aligned}
\frac{\partial g(L-}{} & \left.x_{1}-x_{2}-x_{3}-\cdots-x_{n-1}\right) \\
\partial x_{1} & -3 A\left(L^{2}-L x_{1}-L x_{2}-\cdots-L x_{n-1}\right. \\
= & -x_{1} L+x_{1}^{2}+x_{1} x_{2}+\cdots+x_{1} x_{n-1} \\
& -x_{2} L+x_{2} x_{1}+x_{2}^{2}+\cdots+x_{2} x_{n-1} \\
& \vdots \\
& \left.\quad-x_{n-1} L+x_{n-1} x_{1}+x_{n-1} x_{2}+\cdots+x_{n-1}^{2}\right) \\
& -2 B L+2 B x_{1}+2 B x_{2}+\cdots+2 B x_{n-1}-C
\end{aligned}
$$

Now adding $\frac{\partial g\left(x_{1}\right)}{\partial x_{1}}$ and $\frac{\partial g\left(L-x_{1}-x_{2}-x_{3}-\cdots-x_{n-1}\right)}{\partial x_{1}}$ together: 


$$
\begin{aligned}
\frac{\partial G(\bar{x})}{\partial x_{1}}= & \frac{\partial g\left(x_{1}\right)}{\partial x_{1}}+\frac{\partial g\left(L-x_{1}-x_{2}-x_{3}-\cdots-x_{n-1}\right)}{\partial x_{1}} \\
= & 3 A x_{1}^{2}+2 B x_{1}+C \\
& -3 A\left(L^{2}-L x_{1}-L x_{2}-\cdots-L x_{n-1}\right. \\
& -x_{1} L+x_{1}^{2}+x_{1} x_{2}+\cdots+x_{1} x_{n-1} \\
& -x_{2} L+x_{2} x_{1}+x_{2}^{2}+\cdots+x_{2} x_{n-1} \\
& \vdots \\
& \left.-x_{n-1} L+x_{n-1} x_{1}+x_{n-1} x_{2}+\cdots+x_{n-1}^{2}\right) \\
& -2 B L+2 B x_{1}+2 B x_{2}+\cdots+2 B x_{n-1}-C
\end{aligned}
$$

After simplifying and factoring the right hand side:

$$
\begin{aligned}
\frac{\partial G(\bar{x})}{\partial x_{1}} & =\frac{\partial g\left(x_{1}\right)}{\partial x_{1}}+\frac{\partial g\left(L-x_{1}-x_{2}-x_{3}-\cdots-x_{n-1}\right)}{\partial x_{1}} \\
& =-3 A\left(L-x_{2}-x_{3}-\cdots-x_{n-1}+\frac{2 B}{3 A}\right)\left(L-2 x_{1}-x_{2}-\cdots-x_{n-1}\right)
\end{aligned}
$$

So there are two solutions for $\frac{\partial G(\bar{x})}{\partial x_{1}}=0$, one is $x_{1}=\frac{{ }^{L-} \sum_{j=2}^{n-1} x_{j}}{2}$ and the other is $\sum_{j=2}^{n-1} x_{j}=L-2 m$. 
So far we have found the solutions to (8), but it would be preferable if they were stated explicitly in terms of the loads on servers. Before proceeding, we give an example of the resulting workload assignments using the solutions from (8).

Suppose that we have three servers and $2 m<L<4 m$, based on (9) and (10) we have the following:

$$
\begin{aligned}
& \frac{\partial G(\bar{x})}{\partial x_{1}}=0 \Rightarrow\left\{\begin{array}{l}
(p) \text { using } 9): x_{1}=\frac{L-x_{2}}{2} \\
(q) \text { using } 10): x_{2}=L-2 m
\end{array}\right. \\
& \frac{\partial G(\bar{x})}{\partial x_{2}}=0 \Rightarrow\left\{\begin{array}{l}
(r) \text { using } 9): x_{2}=\frac{L-x_{1}}{2} \\
(s) \text { using } 10): x_{1}=L-2 m
\end{array}\right.
\end{aligned}
$$

For $\frac{\partial G(\bar{x})}{\partial x_{\ell}}=0, \ell=1,2$ for each $x_{\ell}$ there are two solutions so overall there are four possible solutions (some solutions may be duplicated). Let us look at all four possible solutions of $\frac{\partial G(\bar{x})}{\partial x_{\ell}}=0, \ell=1,2$ and find the load on each of the three servers: 
- $(p),(r): x_{1}=\frac{L-x_{2}}{2}$ and $x_{2}=\frac{L-x_{1}}{2}$ :

$$
\begin{aligned}
x_{1}=\frac{L-x_{2}}{2} & \Rightarrow 2 x_{1}=L-x_{2} \\
x_{2}=\frac{L-x_{1}}{2} & \Rightarrow 2 x_{2}=L-x_{1} \\
& \Rightarrow x_{1}=x_{2}=\frac{L}{3} \\
& \Rightarrow x_{3}=L-x_{1}-x_{2}=\frac{L}{3}
\end{aligned}
$$

- $(p),(s): x_{1}=\frac{L-x_{2}}{2}$ and $x_{1}=L-2 m$ :

$$
\begin{aligned}
x_{1}=\frac{L-x_{2}}{2} & , x_{1}=L-2 m \\
\Rightarrow x_{2} & =4 m-L \\
\Rightarrow x_{3} & =L-2 m=x_{1}
\end{aligned}
$$

- $(q),(r): x_{2}=L-2 m$ and $x_{2}=\frac{L-x_{1}}{2}$

$$
\begin{array}{r}
\quad x_{1}=4 m-L \\
x_{2}=L-2 m \\
x_{3}=L-2 m=x_{2}
\end{array}
$$

- $(q),(s): x_{2}=L-2 m$ and $x_{1}=L-2 m$

$$
x_{3}=L-x_{1}-x_{2}=4 m-L
$$


All of these four possibilities are equivalent to two different configurations, one configuration is one server with load $x=4 m-L$ and the remaining two servers with load $x=L-2 m$, the second configuration is assigning the load equally to three servers so each of the servers has load $x_{i}=\frac{L}{3}$. Both of these two configurations follow the format of configurations $(a)$ and $(b)$ described in Lemma 4 for $n=3$ and $i=1$.

With this example in mind, let us use Lemma 4 to convert (9) and (10) to corresponding loads on servers. In the following lemmas, we will assume $L \neq n m$. This is essentially without loss of generality, as this is just one specific value of $L$.

Lemma 4. Suppose $L \neq n m$. The solutions to (8) are equivalent to the following workload assignments:

(a) $n$ servers are assigned equal load $x=\frac{L}{n}$ (so the total loss would be $\left.n g\left(\frac{L}{n}\right)\right)$

(b) For $1 \leq i<\min \left(\frac{n}{2}, \frac{L}{2 m}, n-\frac{L}{2 m}\right),(n-i)$ servers have load $\frac{L-2 i m}{n-2 i}$ and $i$ servers have load $\frac{2 m(n-i)-L}{n-2 i}$. In this case the total loss would be

$$
(n-i) g\left(\frac{L-2 i m}{n-2 i}\right)+i g\left(\frac{2 m(n-i)-L}{n-2 i}\right)
$$




\section{Proof.}

For a solution to (8) using (9) or (10), there are three possibilities:

(I) Picking (9) for each $x_{\ell}$

(II) Using (9) for $n-i-1$ of the loads $x_{\ell}$, and for the remaining servers using 10

(III) Using (10) for each of the loads $x_{\ell}$

- Analysing $(\mathrm{I})$.

If for each $x_{\ell}$ we pick 99 as the solution of $\frac{\partial G(\bar{x})}{\partial x_{\ell}}=0$, we have the following: 


$$
\begin{gathered}
x_{1}=\frac{L-\sum_{j=2}^{n-1} x_{j}}{2} \\
x_{2}=\frac{L-\sum_{j=1, j \neq 2}^{n-1} x_{j}}{2} \\
\vdots \\
x_{n-1}=\frac{}{L-\sum_{j=1}^{n-2} x_{j}}
\end{gathered}
$$

Let us analyse the first two equations and find the relation between $x_{1}$ and $x_{2}$ :

$$
\begin{gathered}
x_{1}=\frac{L-\left(x_{2}+x_{3}+\cdots+x_{n-1}\right)}{2} \Rightarrow 2 x_{1}=L-\left(x_{2}+x_{3}+\cdots+x_{n-1}\right) \\
x_{2}=\frac{L-\left(x_{1}+x_{3}+\cdots+x_{n-1}\right)}{2} \Rightarrow 2 x_{2}=L-\left(x_{1}+x_{3}+\cdots+x_{n-1}\right) \\
\Rightarrow 2 x_{2}-2 x_{1}=-x_{1}+x_{2} \\
\Rightarrow x_{2}=x_{1}
\end{gathered}
$$


It is not difficult to see that the proof above is identical for all of the other pairs $x_{i}$ and $x_{j}$, so we can conclude that $x_{1}=x_{2}=\cdots=x_{n-1}$.

By adding the equations in (11) together, we get the following:

$$
\begin{aligned}
\sum_{\ell=1}^{n-1} x_{\ell} & =\frac{(n-1) L-(n-2) \sum_{\ell=1}^{n-1} x_{\ell}}{2} \\
\Rightarrow 2 \sum_{\ell=1}^{n-1} x_{\ell} & =(n-1) L-(n-2) \sum_{\ell=1}^{n-1} x_{\ell} \\
\Rightarrow & \sum_{\ell=1}^{n-1} x_{\ell}=\frac{n-1}{n} L
\end{aligned}
$$

As $x_{1}=x_{2}=\cdots=x_{n-1}$,

$$
\begin{aligned}
\sum_{\ell=1}^{n-1} x_{\ell}=(n-1) x_{1} & =\frac{n-1}{n} L \\
& \Rightarrow x_{1}=\frac{L}{n}
\end{aligned}
$$

So:

$$
x_{1}=x_{2}=\cdots=x_{n-1}=\frac{L}{n}
$$


We can also find that $x_{n}=\frac{L}{n}$ by using $\sum_{\ell=1}^{n} x_{\ell}=L$.

So (I) results in assigning the load equally on the servers, which is configuration (a) in Lemma 4 .

- Analysing (II)

Another possible solution for $\frac{\partial G(\bar{x})}{\partial x_{\ell}}=0, \ell=1,2, \ldots, n-1$ is choosing $i$ server loads using (10) and $(n-i-1)$ loads using (9). Let us first start with the $(n-i-1)$ equations of $(9)$ :

$$
\begin{gathered}
x_{1}=\frac{L-\sum_{j=2}^{n-1} x_{j}}{2} \\
\vdots \\
x_{n-i-1}=\frac{L-\sum_{j=1, j \neq n-i-1}^{n-1} x_{j}}{2}
\end{gathered}
$$

Similar to the derivation for (I), we can prove: 


$$
x_{1}=x_{2}=\cdots=x_{n-i-1}
$$

On the other hand, there are the $i$ loads chosen according to (10):

$$
\sum_{j=1, j \neq n-i}^{n-1} x_{j}=L-2 m
$$

$$
\sum_{j=1, j \neq n-2}^{n-1} x_{j}=L-2 m
$$

$$
\sum_{j=1}^{n-2} x_{j}=L-2 m
$$

By forming the difference between each pair of the the equations above, we can conclude:

$$
x_{n-i}=x_{n-i+1}=\cdots=x_{n-1}
$$

Substituting $x_{1}$ for all $x_{j}, j=1, \ldots, n-i-1$ and $x_{n-1}$ for all $x_{j}, j=$ 


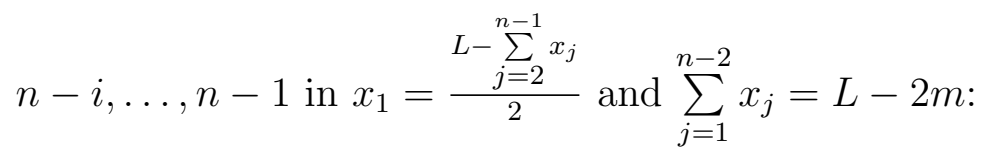

(i)

$$
\begin{aligned}
& x_{1}= \frac{L-\sum_{j=2}^{n-1} x_{j}}{2} \\
&= \frac{L-\left((n-i-2) x_{1}+i x_{n-1}\right)}{2} \Rightarrow \\
&(n-i) x_{1}+i x_{n-1}=L
\end{aligned}
$$

(ii)

$$
\begin{gathered}
\sum_{j=1}^{n-2} x_{j}=L-2 m \Rightarrow \\
(n-i-1) x_{1}+(i-1) x_{n-1}=L-2 m
\end{gathered}
$$

Multiplying 13 by $\frac{i}{i-1}$ then subtracting from 12 we have: 


$$
\begin{gathered}
\left((n-i) x_{1}+i x_{n-1}\right)-\frac{i}{i-1}\left((n-i-1) x_{1}+(i-1) x_{n-1}\right)=L-\frac{i}{i-1}(L-2 m) \\
(n-i) x_{1}-\frac{i(n-i-1)}{i-1} x_{1}=L-\frac{i}{i-1}(L-2 i m) \\
x_{1}=\frac{L-2 i m}{n-2 i}
\end{gathered}
$$

So:

$$
x_{1}=x_{2}=\cdots=x_{n-i-1}=\frac{L-2 i m}{n-2 i}
$$

Substituting $x_{1}$ in 13 :

$$
\begin{gathered}
(n-i-1)\left(\frac{L-2 i m}{n-2 i}\right)+(i-1) x_{n-1}=L-2 m \\
x_{n-1}=\frac{2 m(n-i)-L}{n-2 i}
\end{gathered}
$$

So:

$$
x_{n-i}=x_{n-i+1}=\cdots=x_{n-1}=\frac{2 m(n-i)-L}{n-2 i}
$$


Now let us find $x_{n}$, by substituting for $x_{1}$ and $x_{n-1}$ in (13):

$$
\begin{gathered}
(n-i-1)\left(\frac{L-2 i m}{n-2 i}\right)+i\left(\frac{2 m(n-i)-L}{n-2 i}\right)+x_{n}=L \\
x_{n}=L-\left(\frac{(n-i-1)(L-2 i m)}{n-2 i}+i \frac{2 m(n-i)-L}{n-2 i}\right) \\
x_{n}=\frac{L-2 i m}{n-2 i}
\end{gathered}
$$

So $x_{n}$ is equal to $x_{1}$ which means we have $n-i$ servers with load $\frac{L-2 i m}{n-2 i}$ and $i$ servers with load $\frac{2 m(n-i)-L}{n-2 i}$ which corresponds to configuration $(b)$ in Lemma 4. Note that the reason that we have the condition $i<\frac{n}{2}$ is to avoid duplicated answers (because if $i>\frac{n}{2}$, by substituting $n-i$ for $i$ in $(n-i) g\left(\frac{L-2 i m}{n-2 i}\right)$, it will be equal to $\left.i g\left(\frac{2 m(n-i)-L}{n-2 i}\right)\right)$.

\section{- Analysing (III)}

The last possibility is choosing 10 for each $x_{\ell}$ in $\frac{\partial G(\bar{x})}{\partial x_{\ell}}=0, \ell=1,2, \cdots, x_{n-1}$ : 


$$
\begin{gathered}
\sum_{j=2}^{n-1} x_{j}=L-2 m \\
\sum_{j=1, j \neq 2}^{n-1} x_{j}=L-2 m \\
\vdots \\
\sum_{j=1}^{n-2} x_{j}=L-2 m
\end{gathered}
$$

Similar to the proofs for (I) and (II), we can conclude $x_{1}=x_{2}=\cdots=x_{n-1}$.

After summing the left and right sides of the equations in (14), we will have:

$$
\begin{gathered}
(n-2) \sum_{j=1}^{n-1} x_{j}=(n-1)(L-2 m) \Rightarrow \\
\sum_{j=1}^{n-1} x_{j}=\frac{(n-1)(L-2 m)}{n-2} .
\end{gathered}
$$

Let us find $x_{n}$ : 


$$
\begin{aligned}
x_{n} & =L-\sum_{j=1}^{n-1} x_{j} \\
& =L-\frac{(n-1)(L-2 m)}{n-2} \\
& =\frac{2 m(n-1)-L}{n-2} .
\end{aligned}
$$

On the other hand, since $x_{1}=x_{2}=\cdots=x_{n-1}$ :

$$
\begin{aligned}
x_{1}=x_{2}=\cdots=x_{n-1} & =\frac{1}{n-1} \sum_{j=1}^{n-1} x_{j} \\
& =\frac{1}{n-1} \frac{(n-1)(L-2 m)}{n-2} \\
& =\frac{L-2 m}{n-2} .
\end{aligned}
$$

So $n-1$ servers have load $\frac{L-2 m}{n-2}$ and one server has load $\frac{2 m(n-1)-L}{n-2}$ which corresponds to configuration (b) in Lemma 4 when $i=1$. 
So we showed that Lemma 4 gives explicit workload assignments for the extrema in Lemma 3. Note that both of these two lemmas are for $n$ servers (i.e. $n$ active servers) and we have $n \leq N$.

Now that we have determined that the set of minima and maxima of $G(\bar{x})$ follow either configuration $(a)$ or configuration $(b)$ in Lemma 4 , we can find which entry is the minimum. In Lemma 5 and Lemma 6 we will show that the minimum follows the format of configuration $(a)$ and not $(b)$. Then, depending on $L$, we will find the minimum of $G(\bar{x})$ using Lemma 7 and Lemma 8. If $L \leq N x_{\max }$, Lemma 7 will show that between all of the possible choices of $n(n \leq N)$ in configuration (a), when $n=\frac{L}{x_{\max }}$ (if $\frac{L}{x_{\max }}$ is an integer) or either $n=\left\lfloor\frac{L}{x_{\max }}\right\rfloor$ or $n=\left\lceil\frac{L}{x_{\max }}\right\rceil, G(\bar{x})$ is minimized for sufficiently large $L$ and if $L>N x_{\text {max }}$, Lemma 8 shows by assigning $L$ equally to all $N$ servers, $G(\bar{x})$ is minimized.

Let us start with Lemma 5 and Lemma 6. Lemma 5 is for the integer case which means when $\frac{L}{x_{\max }}$ is an integer and Lemma 6 is for when $\frac{L}{x_{\max }}$ is not an integer. Recall that in Lemma 3, $n \geq 3$ so $L-2 m>0$ in (10), on the other hand $L=\alpha m$, so $\alpha>2$.

Lemma 5. If $\frac{L}{x_{\max }}$ is an integer, the total loss of configuration (a) for $n=$ $\frac{L}{x_{\max }}$ is smaller than the loss of configuration (b) for any $n$ and $i$. 


\section{Proof.}

We are going to prove inequality (15) below:

$$
(n-i) g\left(\frac{L-2 i m}{n-2 i}\right)+i g\left(\frac{(n-i) 2 m-L}{n-2 i}\right)-K g\left(\frac{L}{K}\right) \geq 0,
$$

where as a reminder, $K=\frac{L}{x_{\max }}$. Now, using (3) to substitute for $g(x)$ :

$$
\begin{aligned}
& (n-i)\left(A\left(\frac{L-2 i m}{n-2 i}\right)^{3}+B\left(\frac{L-2 i m}{n-2 i}\right)^{2}+C\left(\frac{L-2 i m}{n-2 i}\right)\right) \\
& +i\left(A\left(\frac{(n-i) 2 m-L}{n-2 i}\right)^{3}+B\left(\frac{(n-i) 2 m-L}{n-2 i}\right)^{2}+C\left(\frac{(n-i) 2 m-L}{n-2 i}\right)\right) \\
& -K\left(A\left(\frac{L}{K}\right)^{3}+B\left(\frac{L}{K}\right)^{2}+C\left(\frac{L}{K}\right)\right) \geq 0
\end{aligned}
$$

By substituting $m$ (see (5)) and simplifying the expression above, we have the following: 


$$
\begin{aligned}
& \frac{1}{(n-2 i)^{2}}\left(A L^{3}-\frac{4 i B^{2}(n-i) L}{3 A}-\frac{8 i n(n-i) B^{3}}{27 A^{2}}\right) \\
& +\frac{1}{(n-2 i)^{2}}\left(B n L^{2}+\frac{8 i B^{2}(n-i) L}{3 A}+\frac{4 i B^{3} n(n-i)}{9 A^{2}}\right)+C L \\
& -\frac{A L^{3}}{K^{2}}-\frac{B L^{2}}{K}-C L \geq 0
\end{aligned}
$$

Multiplying both sides by $(n-2 i)^{2}$ and using (6) to substitute for $L$ :

$$
\begin{aligned}
& \frac{-\alpha^{3} B^{3}}{27 A^{2}}-\frac{8 i B^{3} n(n-i)}{27 A^{2}}-\frac{8 i \alpha B^{3}(n-i)}{9 A^{2}}-\frac{\alpha^{2} B^{3}(n-2 i)^{2}}{9 A^{2} K} \\
& \geq \frac{-4 \alpha B^{3} i(n-i)}{9 A^{2}}-\frac{\alpha^{2} B^{3} n}{9 A^{2}}-\frac{4 i B^{3} n(n-i)}{9 A^{2}}-\frac{\alpha^{3} B^{3}(n-2 i)^{2}}{27 A^{2} K^{2}}
\end{aligned}
$$

Multiplying both sides by $-\frac{27 A^{2}}{B^{3}}\left(\right.$ remember $B<0$ so $\left.-\frac{27 A^{2}}{B^{3}}>0\right)$ :

$$
\alpha^{3}\left(1-\frac{(n-2 i)^{2}}{K^{2}}\right)+\alpha^{2}\left(\frac{3(n-2 i)^{2}}{K}-3 n\right)+12 i(n-i) \alpha-4 i n(n-i) \geq 0
$$


Before going ahead, let us simplify $K$, using (4) and (6):

$$
K=\frac{L}{x_{\max }}=\frac{\frac{-B}{3 A} \alpha}{\frac{-B}{2 A}}=\frac{2 \alpha}{3}
$$

After substituting $K$ in (16) and simplifying, we have the following:

$$
\alpha^{3}+\frac{9 n^{2} \alpha}{4}+3 i n \alpha+4 i^{2} n \geq 3 i^{2} \alpha+3 \alpha^{2} n+4 i n^{2}
$$

Denote the left hand side by $c(i)$ and the right hand side by $d(i)$ so we are going to prove that $c(i) \geq d(i)$ in the interval $1 \leq i<\min \left(\frac{n}{2}, \frac{L}{2 m}, n-\frac{L}{2 m}\right)$ (the boundaries of the interval follow from the definition of configuration (b)). Since $c(i)$ and $d(i)$ are continuous functions, if in the entire interval of $i, \frac{\partial c(i)}{\partial i} \geq \frac{\partial d(i)}{\partial i}$ and $c(1) \geq d(1)$ then $c(i) \geq d(i)$ in the entire interval, also if in the entire interval $\frac{\partial c(i)}{\partial i} \leq \frac{\partial d(i)}{\partial i}$ but at the end of the interval of $i$ (let us call it $w), c(w) \geq d(w)$ then again $c(i) \geq d(i)$ in the entire interval. Otherwise, it contradicts the fact that $\frac{\partial c(i)}{\partial i}<\frac{\partial d(i)}{\partial i}$. With this in mind, let us calculate $\frac{\partial c(i)}{\partial i}, \frac{\partial d(i)}{\partial i}$ and $\frac{\partial c(i)}{\partial i}-\frac{\partial d(i)}{\partial i}$ : 


$$
\begin{gathered}
\frac{\partial c(i)}{\partial i}=3 n \alpha+8 i n \\
\frac{\partial d(i)}{\partial i}=6 i \alpha+4 n^{2} \\
\frac{\partial c(i)}{\partial i}-\frac{\partial d(i)}{\partial i}=3 n \alpha+8 i n-\left(6 i \alpha+4 n^{2}\right)=(3 \alpha-4 n)(n-2 i)
\end{gathered}
$$

Since $i<\frac{n}{2}$ (remember $\left.1 \leq i<\min \left(\frac{n}{2}, \frac{L}{2 m}, n-\frac{L}{2 m}\right)\right)$, the second factor is always positive so the sign of the first factor $3 \alpha-4 n$ determines the sign of the entire expression. So we have two possibilities:

- If $3 \alpha-4 n \geq 0$ then $\frac{\partial c(i)}{\partial i} \geq \frac{\partial d(i)}{\partial i}$. In this case, as explained earlier we need to check if $c(1)>d(1)$ then $c(i) \geq d(i)$ over the entire range of $i$.

$$
\begin{aligned}
& c(1)=\alpha^{3}+\frac{9 n^{2} \alpha}{4}+3 n \alpha+4 n \\
& d(1)=3 \alpha+3 \alpha^{2} n+4 n^{2}
\end{aligned}
$$

We can show $c(1)>d(1)$ by using the fact that $\alpha>2$. We need to show the inequality below holds for $\alpha>2$ :

$$
c(1)-d(1)=\left(\frac{9 \alpha}{4}-4\right) n^{2}+\left(3 \alpha+4-3 \alpha^{2}\right) n+\alpha^{3}-3 \alpha \geq 0
$$

If we show that $\left(3 \alpha+4-3 \alpha^{2}\right)^{2}-4\left(\frac{9 \alpha}{4}-4\right)\left(\alpha^{3}-3 \alpha\right)<0$ and $\left(\frac{9 \alpha}{4}-4\right)>0$ 
then (19) holds. For the left side of the first of the two inequalities:

$$
\left(3 \alpha+4-3 \alpha^{2}\right)^{2}-4\left(\frac{9 \alpha}{4}-4\right)\left(\alpha^{3}-3 \alpha\right)=-2(\alpha-2)^{3}
$$

Since $\alpha>2$ then $-2(\alpha-2)^{3}<0$. Also because $\alpha>2$ we also have $\left(\frac{9 \alpha}{4}-4\right)>0$ so $c(1)>d(1)$.

- If $3 \alpha-4 n<0$ then $\frac{\partial c(i)}{\partial i}<\frac{\partial d(i)}{\partial i}$. In this case, we need to find the values of $c(i)$ and $d(i)$ at the end of the interval. By substituting $m$ and $L$ in the boundaries of $i$ (using (5) and (6)), the interval would be $1 \leq i<\min \left(\frac{n}{2}, \frac{\alpha}{2}, n-\frac{\alpha}{2}\right)$. Since each of $\frac{n}{2}, \frac{\alpha}{2}$ and $n-\frac{\alpha}{2}$ could be the end of the interval, we will check these three cases individually.

- If $\min \left(\frac{\alpha}{2}, \frac{n}{2}, n-\frac{\alpha}{2}\right)=\frac{\alpha}{2}$ :

$$
\begin{gathered}
c\left(\frac{\alpha}{2}\right)=\alpha^{3}+\frac{5}{2} \alpha^{2} n+\frac{9 \alpha n^{2}}{4} \\
d\left(\frac{\alpha}{2}\right)=\frac{3 \alpha^{3}}{4}+3 \alpha^{2} n+2 \alpha n^{2} \\
c\left(\frac{\alpha}{2}\right)-d\left(\frac{\alpha}{2}\right)=\frac{\alpha^{3}}{4}-\frac{\alpha^{2} n}{2}+\frac{\alpha n^{2}}{4}=\frac{1}{4}(\alpha-n)^{2} \alpha
\end{gathered}
$$


So $c\left(\frac{\alpha}{2}\right) \geq d\left(\frac{\alpha}{2}\right)$ holds if $\alpha>0$ and recall that $\alpha>2$. So if $\frac{\alpha}{2}$ is the minimum, at the end of the interval of $i, c(i) \geq d(i)$.

- If $\min \left(\frac{\alpha}{2}, \frac{n}{2}, n-\frac{\alpha}{2}\right)=\frac{n}{2}$ :

The inequalities $\frac{n}{2} \leq \frac{\alpha}{2}$ and $\frac{n}{2} \leq n-\frac{\alpha}{2}$ result in $n=\alpha$. By substituting $n=\alpha$ and $i=\frac{n}{2}$ in $c(i)$ and $d(i)$, we have:

$$
c\left(\frac{n}{2}\right)=d\left(\frac{n}{2}\right)=\frac{23}{4} n^{3}
$$

- If $\min \left(\frac{\alpha}{2}, \frac{n}{2}, n-\frac{\alpha}{2}\right)=n-\frac{\alpha}{2}$ :

$$
c\left(n-\frac{\alpha}{2}\right)-d\left(n-\frac{\alpha}{2}\right)=\frac{1}{4} \alpha(n-\alpha)^{2}
$$

If $\alpha>0$ then $c\left(n-\frac{\alpha}{2}\right)-d\left(n-\frac{\alpha}{2}\right) \geq 0$. Recall that $\alpha>2$ so $c\left(n-\frac{\alpha}{2}\right) \geq d\left(n-\frac{\alpha}{2}\right)$.

In all of the cases above, $c(w) \geq d(w)$, which means if $3 \alpha-4 n<0, c(i) \geq d(i)$. In conclusion if $\frac{L}{x_{\max }}$ is an integer and not greater than $N$ then inequality (18), the simplified version of inequality (15), holds.

Note that the proof of this lemma can be done in a simpler way but since we need part of the calculations above for the next lemma, we used the 
proof above. The simpler proof of Lemma 5 follows from the fact that $G(\bar{x}) \geq L\left(1-f\left(x_{\max }\right)\right)$ and this lower bound can be achieved when $\frac{L}{x_{\max }}$ is an integer so we can pick $\frac{L}{x_{\max }}$ servers and assign $x_{\max }$ to each server. Now let us analyse the case that $\frac{L}{x_{\max }}$ is not an integer.

Lemma 6. If $\frac{L}{x_{\max }}$ is not an integer and $L$ is sufficiently large, the total loss of configuration (a) for $n=\left\lfloor\frac{L}{x_{\max }}\right\rfloor$ or $n=\left\lceil\frac{L}{x_{\max }}\right\rceil$ is smaller than the total loss of any configuration (b) for any $n$ and $i$.

\section{Proof.}

If $K$ (remember $K=\frac{2 \alpha}{3}$ from (17)) is not an integer then we use its ceiling or floor function so we have to prove inequality (15) by using $K_{1}=$ $\left\lfloor\frac{2 \alpha}{3}\right\rfloor=\frac{2 \alpha}{3}-\epsilon_{1}$ and $K_{2}=\left\lceil\frac{2 \alpha}{3}\right\rceil=\frac{2 \alpha}{3}+\epsilon$ (see Table 1 ) instead of $K$. We will show the result for $K_{2}$, the proof for $K_{1}$ is similar. First let us substitute $K_{2}=\left\lceil\frac{2 \alpha}{3}\right\rceil=\frac{2 \alpha}{3}+\epsilon$ in inequality 16 and simplify it:

$$
\begin{aligned}
& \alpha^{3}\left(\left(\frac{2 \alpha}{3}+\epsilon\right)^{2}+4 i n\right)+\alpha^{2}\left(3 n^{2}+12 i^{2}\right)\left(\frac{2 \alpha}{3}+\epsilon\right)+12 i \alpha\left(\frac{2 \alpha}{3}+\epsilon\right)^{2} n+4 i^{2} n\left(\frac{2 \alpha}{3}+\epsilon\right)^{2} \\
& \geq \alpha^{3}\left(n^{2}+4 i^{2}\right)+\alpha^{2}\left(\frac{2 \alpha}{3}+\epsilon\right)\left(12 i n+3 n\left(\frac{2 \alpha}{3}+\epsilon\right)\right)+12 i^{2} \alpha\left(\frac{2 \alpha}{3}+\epsilon\right)^{2}+4 i n^{2}\left(\frac{2 \alpha}{3}+\epsilon\right)^{2}
\end{aligned}
$$

Let us expand the inequality such that terms depending on $\epsilon$ (the fractional 
parts) are isolated:

$$
\begin{aligned}
& \frac{16 i^{2} n \alpha^{2}}{9}+\frac{4 i n \alpha^{3}}{3}+n^{2} \alpha^{3}+\frac{4 \alpha^{5}}{9}+\alpha^{3}\left(\epsilon^{2}+\frac{4 \alpha \epsilon}{3}\right)+\epsilon\left(3 n^{2} \alpha^{2}+12 i^{2} \alpha^{2}\right) \\
& +12 i \alpha n\left(\epsilon^{2}+\frac{4 \alpha \epsilon}{3}\right)+4 i^{2} n\left(\epsilon^{2}+\frac{4 \alpha \epsilon}{3}\right) \\
& \geq \frac{4 i^{2} \alpha^{3}}{3}+\frac{16 i n^{2} \alpha^{2}}{9}+\frac{4 n \alpha^{4}}{3}+2 \alpha^{3} n \epsilon+\epsilon \alpha^{2}(12 i n+2 n \alpha+3 n \epsilon) \\
& +12 i^{2} \alpha\left(\epsilon^{2}+\frac{4 \alpha \epsilon}{3}\right)+4 i n^{2}\left(\epsilon^{2}+\frac{4 \alpha \epsilon}{3}\right)
\end{aligned}
$$

Let us call the part that is independent of $\epsilon$ in the left side of the inequality $c^{\prime}(i)$ and the remaining part $e(i, \epsilon)$ and in the right side let us call them $d^{\prime}(i)$ and $f(i, \epsilon)$ in order, so the inequality above is $c^{\prime}(i)+e(i, \epsilon) \geq d^{\prime}(i)+f(i, \epsilon)$ so $e(i, \epsilon)$ and $f(i, \epsilon)$ would be:

$$
\begin{aligned}
e(i, \epsilon)= & \alpha^{3}\left(\epsilon^{2}+\frac{4 \alpha \epsilon}{3}\right)+\epsilon\left(3 n^{2} \alpha^{2}+12 i^{2} \alpha^{2}\right) \\
& +12 i \alpha n\left(\epsilon^{2}+\frac{4 \alpha \epsilon}{3}\right)+4 i^{2} n\left(\epsilon^{2}+\frac{4 \alpha \epsilon}{3}\right)
\end{aligned}
$$




$$
\begin{aligned}
f(i, \epsilon)= & 2 \alpha^{3} n \epsilon+\epsilon \alpha^{2}(12 i n+2 n \alpha+3 n \epsilon) \\
& +12 i^{2} \alpha\left(\epsilon^{2}+\frac{4 \alpha \epsilon}{3}\right)+4 i n^{2}\left(\epsilon^{2}+\frac{4 \alpha \epsilon}{3}\right)
\end{aligned}
$$

Let us first consider just the integer parts $\left(c^{\prime}(i)\right.$ and $\left.d^{\prime}(i)\right)$, by multiplying them by $\frac{9}{4 \alpha^{2}}$ we will get $c(i)$ and $d(i)$ in $(18)$ in Lemma 5 and we already proved that $c(i) \geq d(i)$ so $c^{\prime}(i) \geq d^{\prime}(i)$.

If we prove $c^{\prime}(i)-d^{\prime}(i) \geq f(i, \epsilon)-e(i, \epsilon)$ then $c^{\prime}(i)+e(i, \epsilon) \geq d^{\prime}(i)+f(i, \epsilon)$. So let us check whether $c^{\prime}(i)-d^{\prime}(i) \geq f(i, \epsilon)-e(i, \epsilon)$ or not. First, let us find $c^{\prime}(i)-d^{\prime}(i)$ :

$$
c^{\prime}(i)-d^{\prime}(i)=\frac{4 \alpha^{4}}{3}\left(\frac{\alpha}{3}-n\right)+\alpha^{3}\left(n^{2}+\frac{4 i n}{3}-\frac{4 i^{2}}{3}\right)+\frac{16 \alpha^{2} i n}{9}(i-n)
$$

We can show $\alpha=\frac{V U(n) n}{m}$ because of the following:

$U(N)=\frac{\sum_{i=1}^{N} u_{i}}{N}, u_{i}$ is the utilization of server $i\left(u_{i}=\frac{L_{i}}{V}, L_{i}\right.$ is the load on server $i$ and $V$ is the capacity of the server). We also have $N U(N)=n U(n)$.

$$
\left\{\begin{array}{l}
L=\sum_{i=1}^{N} L_{i}=V \sum_{i=1}^{N} u_{i}=V U(n) n \\
L=\alpha m
\end{array}\right.
$$




$$
\Rightarrow \alpha m=V U(n) n \Rightarrow \alpha=\frac{V U(n) n}{m}
$$

Now by substituting $\alpha=\frac{V U(n) n}{m}$ in $c^{\prime}(i)-d^{\prime}(i), e(i, \epsilon)$ and $f(i, \epsilon)$ :

$$
\begin{aligned}
c^{\prime}(i)-d^{\prime}(i)= & \frac{4}{3}\left(\frac{V U(n) n}{m}\right)^{4}\left(\frac{V U(n) n}{3 m}-n\right)+\left(\frac{V U(n) n}{m}\right)^{3}\left(n^{2}+\frac{4}{3} i n-\frac{4 i^{2}}{3}\right) \\
& +\frac{16}{9}\left(\frac{V U(n) n}{m}\right)^{2} i n(i-n) \\
= & \frac{4}{9}\left(\frac{V U(n)}{m}\right)^{5} n^{5}-\frac{4}{3}\left(\frac{V U(n)}{m}\right)^{4} n^{5}+\left(\frac{V U(n)}{m}\right)^{3} n^{5}+\frac{4}{3}\left(\frac{V U(n)}{m}\right)^{3} i n^{4} \\
& -\frac{4}{3}\left(\frac{V U(n)}{m}\right)^{3} i^{2} n^{3}+\frac{16}{9}\left(\frac{V U(n)}{m}\right)^{2} i^{2} n^{3}-\frac{16}{9}\left(\frac{V U(n)}{m}\right)^{2} i n^{4} \\
= & n^{5}\left(\frac{V U(n)}{m}\right)^{3}\left(\frac{2 V U(n)}{3 m}-1\right)^{2}+n^{4} \frac{4 i}{3}\left(\frac{V U(n)}{m}\right)^{2}\left(\frac{V U(n)}{m}-\frac{4}{3}\right) \\
& +n^{3} \frac{4 i^{2}}{3}\left(\frac{V U(n)}{m}\right)^{2}\left(\frac{4}{3}-\frac{V U(n)}{m}\right)^{4}
\end{aligned}
$$




$$
\begin{aligned}
e(i, \epsilon)= & \left(\frac{V U(n) n}{m}\right)^{3} \epsilon^{2}+\frac{4}{3}\left(\frac{V U(n) n}{m}\right)^{4} \epsilon+\epsilon\left(3 n^{2}\left(\frac{V U(n) n}{m}\right)^{2}+12 i^{2}\left(\frac{V U(n) n}{m}\right)^{2}\right) \\
& +12 i\left(\frac{V U(n) n}{m}\right) n \epsilon^{2}+16 i\left(\frac{V U(n) n}{m}\right)^{2} n \epsilon+4 i^{2} n \epsilon^{2}+\frac{16}{3} i^{2}\left(\frac{V U(n) n}{m}\right) n \epsilon \\
= & \left(\frac{V U(n)}{m}\right)^{3} n^{3} \epsilon^{2}+\frac{4}{3}\left(\frac{V U(n)}{m}\right)^{4} n^{4} \epsilon+\epsilon\left(3 n^{4}\left(\frac{V U(n)}{m}\right)^{2}+12 i^{2} n^{2}\left(\frac{V U(n)}{m}\right)^{2}\right) \\
& +12 i\left(\frac{V U(n)}{m}\right) n^{2} \epsilon^{2}+16 i\left(\frac{V U(n)}{m}\right)^{2} n^{3} \epsilon+4 i^{2} n \epsilon^{2}+\frac{16}{3} i^{2}\left(\frac{V U(n)}{m}\right) n^{2} \epsilon
\end{aligned}
$$

$$
\begin{aligned}
f(i, \epsilon)= & 2\left(\frac{V U(n)}{m}\right)^{3} n^{4} \epsilon+12 i n^{3}\left(\frac{V U(n)}{m}\right)^{2} \epsilon+2 n^{4}\left(\frac{V U(n)}{m}\right)^{3} \epsilon+3 n^{3}\left(\frac{V U(n)}{m}\right)^{2} \epsilon^{2} \\
& +12 i^{2} n\left(\frac{V U(n)}{m}\right) \epsilon^{2}+16 i^{2} n^{2}\left(\frac{V U(n)}{m}\right)^{2} \epsilon+4 i n^{2} \epsilon^{2}+\frac{16}{3} i n^{3}\left(\frac{V U(n)}{m}\right) \epsilon
\end{aligned}
$$

The maximum power of $n$ in $c^{\prime}(i)-d^{\prime}(i)$ is 5 and its coefficient is positive but the maximum power of $n$ in both $e(i, \epsilon)$ and $f(i, \epsilon)$ is 4 so for sufficiently large $n, c^{\prime}(i)-d^{\prime}(i) \geq f(i, \epsilon)-e(i, \epsilon)$.

The proof above holds for sufficiently large $L$ but in general we have not 
been able to find a lower bound for $L$ or a combination of $L, \epsilon$ and $i$ such that $c^{\prime}(i)-d^{\prime}(i) \geq f(i, \epsilon)-e(i, \epsilon)$ is violated. We believe this inequality holds for all $L, \epsilon$ and $i$ so there is no need to take $L$ sufficiently large but we have been unable to prove it. So we already proved that the minimum of $G(\bar{x})$ is following the format of configuration $(a)$, in the following lemma we will find the minimum.

Lemma 7. Between all configurations following the format of (a), the one with either $n=\left\lfloor\frac{L}{x_{\max }}\right\rfloor$ or $n=\left\lceil\frac{L}{x_{\max }}\right\rceil$ is the minimum of $G(\bar{x})$

\section{Proof.}

By assigning load $L$ equally to $K_{1}=\left\lfloor\frac{L}{x_{\max }}\right\rfloor$ or $K_{2}=\left\lceil\frac{L}{x_{\max }}\right\rceil$ servers, the load on each server is the closest possible load to $x_{\max }$. If the load is assigned to $K_{1}$ servers, the load on each server is on the right side of $x_{\max }$ in the efficiency curve (Fig. 11) and if the load is assigned to $K_{2}$ servers, the load on each server is on the left side of $x_{\max }$. Let $K^{\prime}$ be an integer not equal to $K_{1}$ or $K_{2}$ and suppose $G\left(\frac{L}{K_{1}}, \cdots, \frac{L}{K_{1}}\right) \leq G\left(\frac{L}{K_{2}}, \cdots, \frac{L}{K_{2}}\right)$ (the proof of the case $G\left(\frac{L}{K_{2}}, \cdots, \frac{L}{K_{2}}\right)<G\left(\frac{L}{K_{1}}, \cdots, \frac{L}{K_{1}}\right)$ is similar to this case). $K^{\prime}$ is either greater than $K_{2}$ (so $\frac{L}{K^{\prime}}$ is less than $\frac{L}{K_{2}}$ and because of the concavity of $f(x)$, $\left.f\left(\frac{L}{K_{2}}\right)>f\left(\frac{L}{K^{\prime}}\right)\right)$ or $K^{\prime}$ is less than $K_{1}\left(\right.$ so $\left.f\left(\frac{L}{K_{1}}\right)>f\left(\frac{L}{K^{\prime}}\right)\right)$. Below, we will show that the lower the efficiency, the higher the total loss and by that we can conclude $G\left(\frac{L}{K_{2}}, \cdots, \frac{L}{K_{2}}\right)<G\left(\frac{L}{K^{\prime}}, \cdots, \frac{L}{K^{\prime}}\right)$ or $G\left(\frac{L}{K_{1}}, \cdots, \frac{L}{K_{1}}\right)<G\left(\frac{L}{K^{\prime}}, \cdots, \frac{L}{K^{\prime}}\right)$ and since $G\left(\frac{L}{K_{1}}, \cdots, \frac{L}{K_{1}}\right) \leq G\left(\frac{L}{K_{2}}, \cdots, \frac{L}{K_{2}}\right)$ then $G\left(\frac{L}{K_{1}}, \cdots, \frac{L}{K_{1}}\right)$ is the mini- 
mum.

Suppose $a^{\prime}$ and $b^{\prime}$ are the load on servers following the format of configuration (a), so we have $\frac{L}{a^{\prime}}$ servers having load $\left(a^{\prime}\right)$ and $\frac{L}{b^{\prime}}$ servers having load $\left(b^{\prime}\right)$. If $\mathbf{a}^{\prime}$ and $\mathbf{b}^{\prime}$ are vectors with identical entries $a^{\prime}$ and $b^{\prime}$ respectively, then if we assume $f\left(a^{\prime}\right)<f\left(b^{\prime}\right)$, we will show $G\left(\mathbf{a}^{\prime}\right)>G\left(\mathbf{b}^{\prime}\right)$ :

$$
\begin{aligned}
f\left(a^{\prime}\right) & <f\left(b^{\prime}\right) \Rightarrow \\
1-f\left(a^{\prime}\right) & >1-f\left(b^{\prime}\right) \Rightarrow \\
\left(\frac{L}{a^{\prime}}\right) a^{\prime}\left(1-f\left(a^{\prime}\right)\right) & >\left(\frac{L}{b^{\prime}}\right) b^{\prime}\left(1-f\left(b^{\prime}\right)\right) \Rightarrow \\
G\left(\mathbf{a}^{\prime}\right) & >G\left(\mathbf{b}^{\prime}\right)
\end{aligned}
$$

So to find the minimum we just need to compare $G\left(\frac{L}{K_{1}}, \cdots, \frac{L}{K_{1}}\right)$ and $G\left(\frac{L}{K_{2}}, \cdots, \frac{L}{K_{2}}\right)$, the one with smaller loss is the minimum of $G(\bar{x})$. In conclusion, the total loss of configuration $(a)$ for $n=\left\lfloor\frac{L}{x_{\max }}\right\rfloor$ or $n=\left\lceil\frac{L}{x_{\max }}\right\rceil$ is smaller than the total loss of any configuration $(b)$.

So by using Lemma 5 to Lemma 7 we proved that the minimum of $G(\bar{x})$ is following the format of configuration $(a)$ when either $n=\left\lceil\frac{L}{x_{\max }}\right\rceil$ or $n=\left\lfloor\frac{L}{x_{\max }}\right\rfloor$ and $L$ is sufficiently large. However, we have yet to consider the case that $\frac{L}{x_{\max }}>N$. The following lemma completes the picture by determining the optimal workload assignment to minimize $G(\bar{x})$ when $\frac{L}{x_{\max }}>N$.

Lemma 8. If $\left\lfloor\frac{L}{x_{\max }}\right\rfloor>N$, assigning the load $L$ equally to all of the servers minimizes $G(\bar{x})$. 


\section{Proof.}

If $K$ is greater than $N$, it means $L>N x_{\max }$. Based on Lemma 4, the minimum is either following the format of configuration $(a)$ or configuration (b). When $K>N$, we are going to show that the minimum is still following configuration $(a)$. Now, suppose that instead of balancing the load across all $N$ servers (configuration $(a)$ ), we assign a load of $x_{1}<\frac{L}{N}$ to $i$ servers and a load $x_{2}>\frac{L}{N}$ to the remaining $N-i$ servers (configuration (b)). So, we have:

$$
i x_{1}+(N-i) x_{2}=L
$$

By using (3) and (7), this yields a total loss of:

$$
G(\bar{x})=i x_{1}\left(1-f\left(x_{1}\right)\right)+(N-i) x_{2}\left(1-f\left(x_{2}\right)\right)
$$

After dividing both sides by $L$, we have:

$$
\frac{G(\bar{x})}{L}=\frac{i x_{1}}{L}\left(1-f\left(x_{1}\right)\right)+\frac{(N-i) x_{2}}{L}\left(1-f\left(x_{2}\right)\right)
$$

Now let $\beta=\frac{i x_{1}}{L}$ and use 21):

$$
\begin{aligned}
\frac{G(\bar{x})}{L} & =\beta\left(1-f\left(x_{1}\right)\right)+(1-\beta)\left(1-f\left(x_{2}\right)\right) \\
& =1-\left(\beta f\left(x_{1}\right)+(1-\beta) f\left(x_{2}\right)\right)
\end{aligned}
$$


By concavity of $f(x)$, we have:

$$
\frac{G(\bar{x})}{L}=1-\left(\beta f\left(x_{1}\right)+(1-\beta) f\left(x_{2}\right)\right) \geq 1-f\left(\beta x_{1}+(1-\beta) x_{2}\right)
$$

Next, by knowing $x_{1}<\frac{L}{N}$, we can bound $\beta$ :

$$
\beta=\frac{i x_{1}}{L}<\frac{i}{N}
$$

Now, from 21):

$$
\frac{i x_{1}}{N}+\frac{(N-i) x_{2}}{N}=\frac{L}{N}
$$

So, as $\beta<\frac{i}{N}$ and $x_{1}<x_{2}$,

$$
\beta x_{1}+(1-\beta) x_{2} \geq \frac{L}{N}
$$

So, as $\frac{L}{N}>x_{\max }$, we have

$$
f\left(\beta x_{1}+(1-\beta) x_{2}\right) \leq f\left(\frac{L}{N}\right) \leq f\left(x_{\max }\right)
$$

This gives: 


$$
1-f\left(\beta x_{1}+(1-\beta) x_{2}\right) \geq 1-f\left(\frac{L}{N}\right)
$$

Then by using (22), we have

$$
\begin{aligned}
& \frac{G(\bar{x})}{L} \geq 1-f\left(\frac{L}{N}\right) \\
& G(\bar{x}) \geq L\left(1-f\left(\frac{L}{N}\right)\right)
\end{aligned}
$$

or

$$
G(\bar{x}) \geq N \frac{L}{N}\left(1-f\left(\frac{L}{N}\right)\right)
$$

The lower bound is achieved by balancing the load over all $N$ servers (Note that the same proof holds for the case of $x_{1}>\frac{L}{N}$ and $\left.x_{2}<\frac{L}{N}\right)$. But we still need to prove that between all of the configurations following the format of (a), assigning $L$ equally to all servers $(N)$ minimizes $G(\bar{x})$. Since $L>N x_{\max }$, the load on each of $N$ servers is greater than $x_{\max }$ in $f(x)$. On the other hand, by assigning the load to $n$ servers, each has load $\frac{L}{n}$ and because $n \leq N$, so $\frac{L}{n}>\frac{L}{N}$ which means $f\left(\frac{L}{n}\right)<f\left(\frac{L}{N}\right)$ (based on the concavity of $\left.f(x)\right)$ and based on 20, $G\left(\frac{L}{n}, \cdots, \frac{L}{n}\right)>G\left(\frac{L}{N}, \cdots, \frac{L}{N}\right)$. So between all configurations following the format of $(a)$, equally assigning the load to $N$ servers minimizes 
$G(\bar{x})$.

So Theorem 1 has been proved. In Chapter 5 we will compare LAWA with two common algorithms (server consolidation and uniform load distribution) and we will show how much power can be saved. 


\section{$5 \quad$ Numerical Results}

In this chapter, we provide some examples of actual servers and calculate their power loss based on server consolidation, uniform workload distribution and LAWA. To find the efficiency function of a server, we looked at hundreds of different servers from different manufacturers, using the data in [23]. In [23], for each server there is a data sheet that includes the efficiency curve and coordinates of just four points of the curve. To find the equation of the curve we could use those four given points and find the function equation using curve fitting in Matlab. However, we preferred to use Engauge Digitizer (a software tool that finds the coordinates of the points of a given curve) to find more points of the efficiency curve. This set of points (the original four plus the additional ones) were then used for curve fitting in Matlab. This way, we have a more precise equation for the server efficiency function. For example, for HP server model number 'D17-310P1A', Fig. 10 is for the efficiency function given in the data sheet and Fig. 11 shows the four given points in the data sheet. First of all, we imported the plot shown in Fig. 10 in Engauge Digitizer, the outcome of the software is the coordinates in Fig. 12. Then we entered the resulting points in Matlab and used curve fitting. Fig. 13 is the approximate curve and function that Matlab produced with an error of at most $0.95 \%$. As you can see, the approximate efficiency

function is $f(x)=-0.11 x^{2}+0.087 x+0.9$. To have $x$ in Watts, we need to substitute $\frac{x}{V}$ in $f(x)$. Note that $V \approx 352$ for this particular server so $f(x)=-0.11\left(\frac{x}{352}\right)^{2}+0.087\left(\frac{x}{352}\right)+0.9$. 


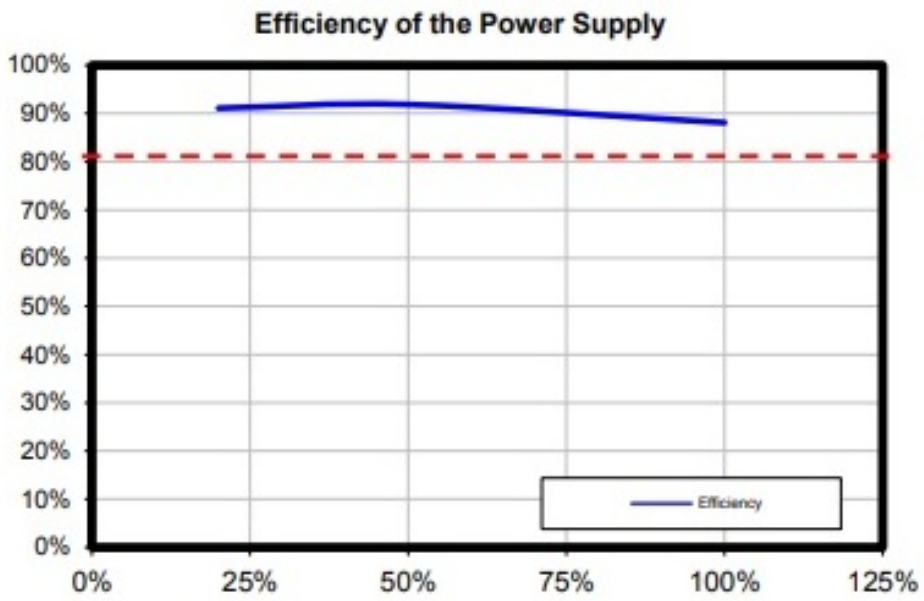

Figure 10: The efficiency curve of HP server model 'D17-310P1A' given in the data sheet

\begin{tabular}{|c|c|c|c|c|}
\hline \multirow{2}{*}{ Load } & Input & DC Terminal Voltage (V)/ DC Load Current (A) & \multirow{2}{*}{$\begin{array}{c}\text { Output } \\
\text { Watts }\end{array}$} & \multirow{2}{*}{ Efficiency } \\
\cline { 3 - 5 } & Watts & 12.1V (cumulative of 12V1, 12V2, etc.) & 31.46 \\
\hline \hline $10 \%$ & 35.96 & $12.26 / 2.57$ & $\mathbf{8 7 . 4 8 \%}$ \\
\hline $20 \%$ & 69.03 & $12.24 / 5.14$ & 62.89 \\
\hline $50 \%$ & 170.51 & $12.18 / 12.86$ & $\mathbf{9 1 . 1 0 \%}$ \\
\hline $100 \%$ & 351.38 & $12.09 / 25.61$ & $\mathbf{9 1 . 8 7 \%}$ \\
\hline
\end{tabular}

Figure 11: Efficiency at different loads of HP server model 'D17-310P1A'

By finding the efficiency function of servers, we can find the corresponding power loss, allowing us to compare the power loss of different algorithms. Here we provide some results for different servers that compare the performance of LAWA to server consolidation and uniform workload distribution. First of all, we have to find the value of $L$ for a given $N$ and $U(N)$ (we will consider $U(N)=0.1,0.5,0.9)$ based on $L=V N U(N)$. Recall that $K=\frac{L}{x_{\max }}$. By looking at the results shown in the tables below, in all of the cases our proposed algorithm has the lowest power loss. To see the amount 


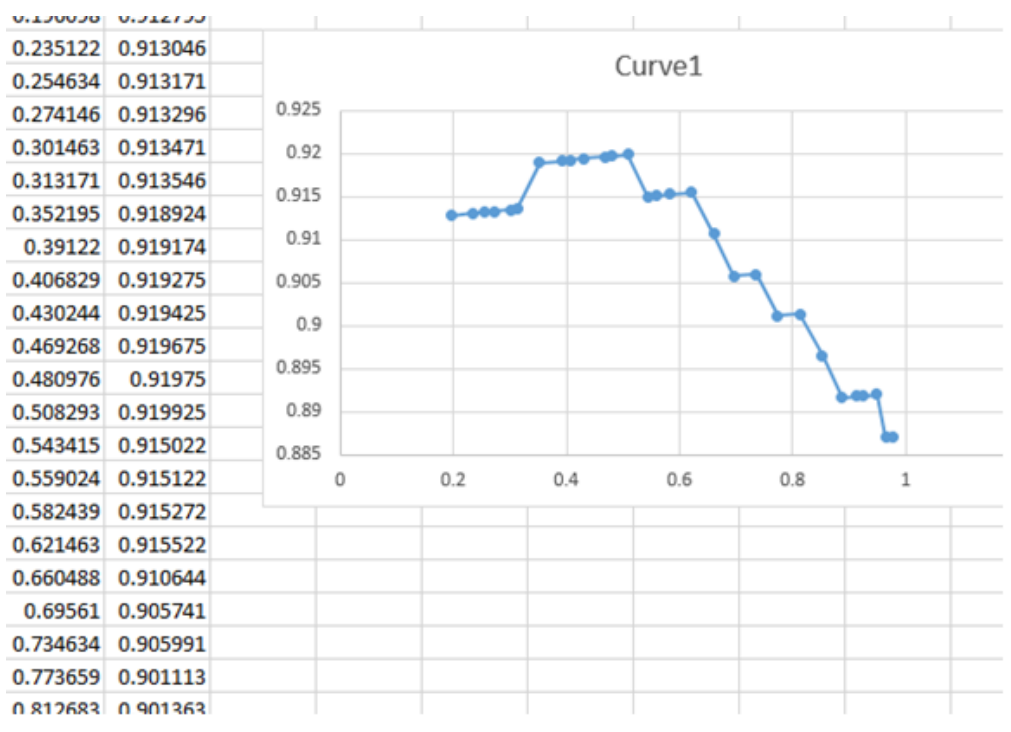

Figure 12: Coordinates produced by Engauge Digitizer for HP server model 'D17-310P1A' 


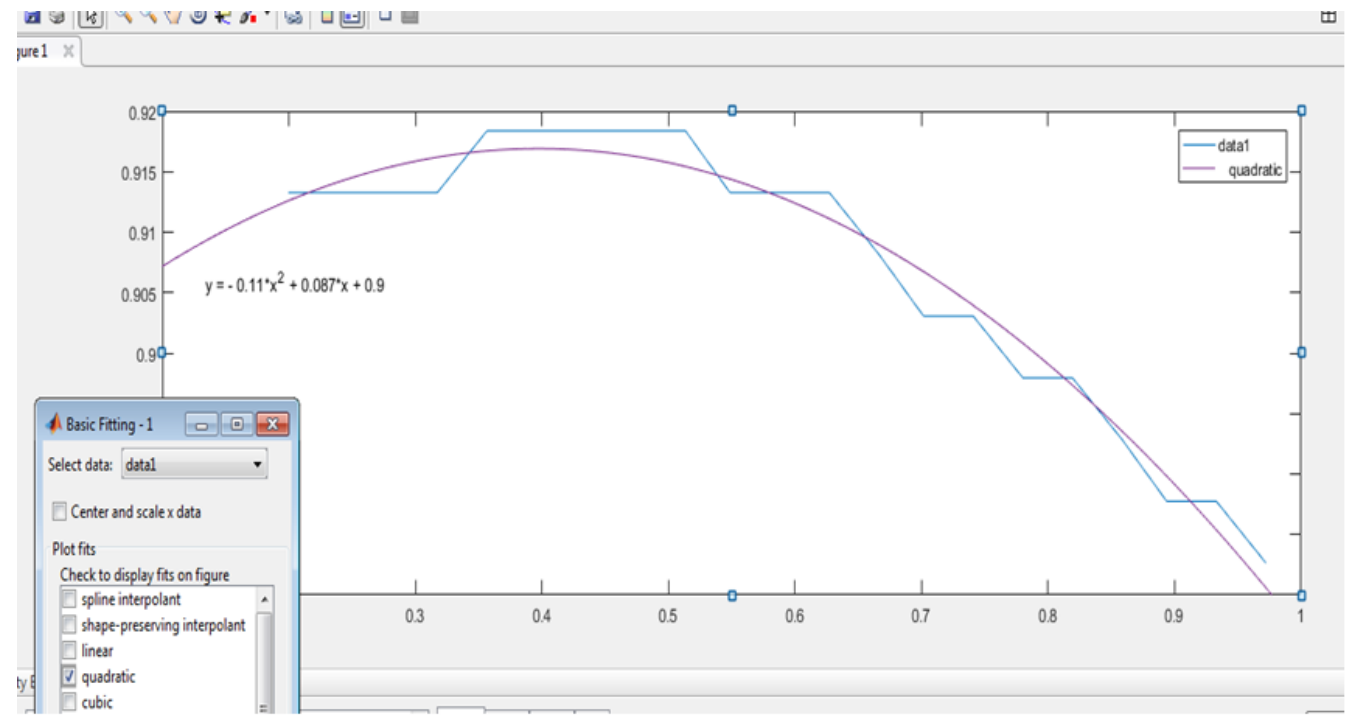

Figure 13: Curve fitting in Matlab for HP server model 'D17-310P1A' 
of power that LAWA is saving, we added the last column to the tables that displays the percentage of the extra power saved by LAWA relative to the algorithm with the maximum power loss .

By looking at Tables 2 and 3 , we see that the rows are similar. The value of columns $K$ through $L A W A$ are proportional to the value of $N$ for a constant $U(N)$ (recall $K=\frac{L}{x_{\max }}=\frac{V N U(N)}{x_{\max }}$, uniform workload distribution is $N g\left(\frac{L}{N}\right)=N g\left(\frac{V N U(N)}{N}\right)=N g(V U(N))$, server consolidation is $\frac{V N U(N)}{V} g(V)$ and LAWA is approximately $\left.\frac{V U(N) N}{x_{\max }} g\left(x_{\max }\right)\right)$. So it makes sense that the amount of saved power in the last column is the same for each value of $U(N)$. Each of the Tables 2 to 7 are from different models from the manufacturers Dell and HP. Independent of the model and the manufacturer, in all of the tables our proposed algorithm reduces the power loss more than uniform workload distribution and server consolidation.

By looking at the results in the tables, while server consolidation is designed to save on idle power consumption, our observations show that the savings are not going to be as large as expected because server consolidation is not taking server power loss into account. Another observation is that LAWA is optimal for the smallest load in Table 3 (the smallest $L$ is when $N=10$ and $U(N)=0.1)$ and this observation is evidence that even for smaller loads LAWA is optimal. 
Table 2: Comparing losses for HP server model D17-310P1A

\begin{tabular}{|c|l|l|l|l|l|l|}
\hline$N$ & $U(N)$ & $K$ & ULDWA & SCWA & LAWA & $\begin{array}{l}\text { Loss } \\
\text { Reduction }\end{array}$ \\
\hline \multirow{2}{*}{10} & 0.1 & 2.5 & 32.52 & 43.29 & 29.145 & $32 \%$ \\
& 0.5 & 12.64 & 147.84 & 216.48 & 147.84 & $31 \%$ \\
& 0.9 & 22.75 & 351.014 & 389.664 & 351.014 & $9.9 \%$ \\
\hline \multirow{2}{*}{100} & 0.1 & 25.2 & 325.24 & 432.96 & 291.456 & $32 \%$ \\
& 0.5 & 126.4 & $1.4784 * 10^{3}$ & $2.1648 * 10^{3}$ & $1.4784 * 10^{3}$ & $31 \%$ \\
& 0.9 & 227.5 & $3.51014 * 10^{3}$ & $3.89664 * 10^{3}$ & $3.51014 * 10^{3}$ & $9.9 \%$ \\
\hline \multirow{2}{*}{1000} & 0.1 & $2.528 * 10^{2}$ & $3.2524 * 10^{3}$ & $4.3296 * 10^{3}$ & $2.91448 * 10^{3}$ & $32 \%$ \\
& 0.5 & $1.264 * 10^{3}$ & $1.4784 * 10^{4}$ & $2.1648 * 10^{4}$ & $1.4784 * 10^{4}$ & $31 \%$ \\
& 0.9 & $2.275 * 10^{3}$ & $3.510144 * 10^{4}$ & $3.8966 * 10^{4}$ & $3.510144 * 10^{4}$ & $9.9 \%$ \\
\hline \multirow{2}{*}{$10^{4}$} & 0.1 & $2.52873 * 10^{3}$ & $3.25248 * 10^{4}$ & $4.3296 * 10^{4}$ & $2.91448 * 10^{4}$ & $32 \%$ \\
& 0.5 & $1.26436 * 10^{4}$ & $1.4784 * 10^{5}$ & $2.1648 * 10^{5}$ & $1.4784 * 10^{5}$ & $31 \%$ \\
& 0.9 & $2.27586 * 10^{4}$ & $3.510144 * 10^{5}$ & $3.89664 * 10^{5}$ & $3.510144 * 10^{5}$ & $9.9 \%$ \\
\hline \multirow{2}{*}{$10^{5}$} & 0.1 & $2.52873 * 10^{4}$ & $3.25248 * 10^{5}$ & $4.3296 * 10^{5}$ & $2.91448 * 10^{5}$ & $32 \%$ \\
& 0.5 & $1.26436 * 10^{5}$ & $1.4784 * 10^{6}$ & $2.1648 * 10^{6}$ & $1.4784 * 10^{6}$ & $31 \%$ \\
& 0.9 & $2.27586 * 10^{5}$ & $3.510144 * 10^{6}$ & $3.89664 * 10^{6}$ & $3.510144 * 10^{6}$ & $9.9 \%$ \\
\hline \multirow{2}{*}{$10^{6}$} & 0.1 & $2.52873 * 10^{5}$ & $3.25248 * 10^{6}$ & $4.3296 * 10^{6}$ & $2.91448 * 10^{6}$ & $32 \%$ \\
& 0.5 & $1.26436 * 10^{6}$ & $1.4784 * 10^{7}$ & $2.1648 * 10^{7}$ & $1.4784 * 10^{7}$ & $31 \%$ \\
& 0.9 & $2.27586 * 10^{6}$ & $3.510144 * 10^{7}$ & $3.89664 * 10^{7}$ & $3.510144 * 10^{7}$ & $9.9 \%$ \\
\hline
\end{tabular}


Table 3: Comparing losses for Dell server model PS-2112-2L

\begin{tabular}{|c|l|l|l|l|l|l|}
\hline$N$ & $U(N)$ & $K$ & ULDWA & SCWA & LAWA & $\begin{array}{l}\text { Loss } \\
\text { Reduction }\end{array}$ \\
\hline \multirow{2}{*}{10} & 0.1 & 2 & 122.58 & 137.17 & 96.64 & $29 \%$ \\
& 0.5 & 10 & 483.21 & 685.85 & 483.21 & $29 \%$ \\
& 0.9 & 18 & 1103.22 & 1234.53 & 1103.22 & $10 \%$ \\
\hline \multirow{2}{*}{100} & 0.1 & 20 & $1.2258 * 10^{3}$ & $1.3717 * 10^{3}$ & $9.664 * 10^{2}$ & $29 \%$ \\
& 0.5 & 100 & $4.8321 * 10^{3}$ & $6.8585 * 10^{3}$ & $4.8321 * 10^{3}$ & $29 \%$ \\
& 0.9 & 180 & $1.10322 * 10^{4}$ & $1.23453 * 10^{4}$ & $1.10322 * 10^{4}$ & $10 \%$ \\
\hline \multirow{2}{*}{1000} & 0.1 & 200 & $1.225801 * 10^{4}$ & $1.3717 * 10^{4}$ & $9.66425 * 10^{3}$ & $29 \%$ \\
& 0.5 & $10^{3}$ & $4.832125 * 10^{4}$ & $6.8585 * 10^{4}$ & $4.832125 * 10^{4}$ & $29 \%$ \\
& 0.9 & $1.8 * 10^{3}$ & $1.10322 * 10^{5}$ & $1.23453 * 10^{5}$ & $1.10322 * 10^{5}$ & $10 \%$ \\
\hline \multirow{2}{*}{$10^{4}$} & 0.1 & $2 * 10^{3}$ & $1.225801 * 10^{5}$ & $1.3717 * 10^{5}$ & $9.66425 * 10^{4}$ & $29 \%$ \\
& 0.5 & $10^{4}$ & $4.832125 * 10^{5}$ & $6.8585 * 10^{5}$ & $4.832125 * 10^{5}$ & $29 \%$ \\
& 0.1 & $2 * 10^{5}$ & $1.225801 * 10^{7}$ & $1.3717 * 10^{7}$ & $9.66425 * 10^{6}$ & $29 \%$ \\
\hline \multirow{2}{*}{$10^{5}$} & 0.1 & $2 * 10^{4}$ & $1.225801 * 10^{6}$ & $1.3717 * 10^{6}$ & $9.66425 * 10^{5}$ & $29 \%$ \\
& 0.5 & $10^{5}$ & $4.8321250 * 10^{6}$ & $6.8585 * 10^{6}$ & $4.832125 * 10^{6}$ & $29 \%$ \\
\hline & 0.9 & $1.8 * 10^{5}$ & $1.10322 * 10^{7}$ & $1.23453 * 10^{7}$ & $1.10322 * 10^{7}$ & $10 \%$ \\
\hline & 0.9 & $1.8 * 10^{6}$ & $1.10322 * 10^{8}$ & $1.23453 * 10^{8}$ & $1.10322 * 10^{8}$ & $10 \%$ \\
\hline
\end{tabular}


Table 4: Comparing losses for Dell server model D750E-S7

\begin{tabular}{|l|l|l|l|l|l|l|}
\hline$N$ & $U(N)$ & $K$ & ULDWA & SCWA & LAWA & $\begin{array}{l}\text { Loss } \\
\text { Reduction }\end{array}$ \\
\hline \multirow{2}{*}{10} & 0.1 & 2.08 & 36.24 & 41.08 & 30.02 & $26 \%$ \\
& 0.5 & 10.4 & 154.05 & 205.4 & 154.05 & $25 \%$ \\
& 0.9 & 18.7 & 337.58 & 369.72 & 337.58 & $8.6 \%$ \\
\hline
\end{tabular}

Table 5: Comparing losses for Dell server model C1100P-00

\begin{tabular}{|l|l|l|l|l|l|l|}
\hline$N$ & $U(N)$ & $K$ & ULDWA & SCWA & LAWA & $\begin{array}{l}\text { Loss } \\
\text { Reduction }\end{array}$ \\
\hline \multirow{2}{*}{10} & 0.1 & 1.5 & 128.92 & 83.72 & 65.78 & $48 \%$ \\
& 0.5 & 7.5 & 328.9 & 418.6 & 300.86 & $28 \%$ \\
& 0.9 & 13.5 & 643.68 & 753.48 & 643.68 & $14 \%$ \\
\hline
\end{tabular}

Table 6: Comparing losses for Dell server model L600E-S0

\begin{tabular}{|l|l|l|l|l|l|l|}
\hline$N$ & $U(N)$ & $K$ & ULDWA & SCWA & LAWA & $\begin{array}{l}\text { Loss } \\
\text { Reduction }\end{array}$ \\
\hline \multirow{2}{*}{10} & 0.1 & 1.68 & 83.61 & 75.02 & 57.97 & $30 \%$ \\
& 0.5 & 8.4 & 289.85 & 375.1 & 285.58 & $23 \%$ \\
& 0.9 & 15.1 & 605.20 & 675.18 & 605.20 & $10 \%$ \\
\hline
\end{tabular}


Table 7: Comparing losses for Dell server model DPSN-300 DB C

\begin{tabular}{|l|l|l|l|l|l|l|}
\hline$N$ & $U(N)$ & $K$ & ULDWA & SCWA & LAWA & $\begin{array}{l}\text { Loss } \\
\text { Reduction }\end{array}$ \\
\hline \multirow{2}{*}{10} & 0.1 & 1.4 & 73.82 & 47.73 & 41.1 & $44 \%$ \\
& 0.5 & 7.2 & 225.91 & 238.69 & 205.77 & $13 \%$ \\
& 0.9 & 13.1 & 394.36 & 429.65 & 394.36 & $8.2 \%$ \\
\hline
\end{tabular}

\section{Conclusions}

In this thesis, we presented a novel approach to minimize the power consumption in data centers by looking at the load distribution on servers. We discussed that due to the power loss being a function of the load, there is the opportunity for significant power savings and the impact of that savings is magnified by the number of servers in today's data centers, as a result server power loss has a significant impact on the total power consumption of data centers. Our proposed algorithm can significantly reduce power consumption compared to the two most common approaches, server consolidation and uniform workload distribution algorithms. Among the six different manufacturers that we studied in Chapter 5, for a large data center, this can translate into savings due to reduced losses of on the order of one million dollars per year. For smaller loads, the policy of loss aware workload assignment is closer to server consolidation but it is not as extreme as the case when one is trying to consolidate the load on the minimum number of servers. One 
potential beneficial side effect is that it is better than server consolidation for latency sensitive workloads. For larger loads LAWA coincides with uniform workload distribution. We also found out that server consolidation may not be saving as much power as expected due to the effects of server power losses.

With respect to future work, this research has developed a novel algorithm to minimize the total power loss of servers in data centers, yet there are significant opportunities for future work. Some possibilities are as follows:

- Considering the idle power of servers (this is the key concern of server consolidation). One reason for not doing this is that the amount of idle power consumed by servers has been decreasing over time and thus the importance of considering this issue has decreased.

- Considering the case that all of the servers in the data center are not necessarily the same, for example when there is a mix of newer (more efficient) and older (less efficient) servers 


\section{$7 \quad$ References}

[1] B. Johnson. "How data centers work." Howstuffworks, URL https:// computer.howstuffworks.com/data-centers1.htm. 22 Jan 2020.

[2] N. Jones. "How to stop data centres from gobbling up the world's electricity." Nature, URL https://www.nature.com/articles/ d41586-018-06610-y. 22 Jan 2020.

[3] A. Shehabi, S. Smith, D. Sartor, R. Brown, M. Herrlin, J. Koomey, E. Masanet, N. Horner, I. Azevedo, W. Lintner. United States data center energy usage report. Tech. Rep. LBNL-1005775, 2016, 1372902.

[4] J. Judge, J. Pouchet, A. Ekbote, S. Dixit. Reducing data center energy consumption. ASHRAE Journal, 50(11):14, 2008.

[5] C. Robertson, J. Romm. Data centers, power, and pollution prevention: Design for business and environmental advantage. The Center for Energy and Climate Solutions, 2002

[6] M. Ala'Anzy, M. Othman. Load balancing and server consolidation in cloud computing environments: A meta-study. IEEE Access, 7:141868141887,2019

[7] N. Yadava, V. K. Mishra, R. K. Chauhan. Design of one-transistor SRAM cell for low power consumption. International Conference on Emerging Trends in Electrical, Electronics and Sustainable Energy Systems (ICETEESES-16), 322-325, 2016 
[8] M. Gupta, S. Grover, S. Singh. A feasibility study for power management in LAN switches. International Conference on Network Protocols (ICNP), 4:361-371, 2004

[9] M. Gupta, S. Singh. Using low-power modes for energy conservation in Ethernet LANs. IEEE INFOCOM 2007-26th IEEE International Conference on Computer Communications, 2451-2455, 2007

[10] S.M. Mirhoseininejad, G. Badawy, and D.G. Down. EAWA: Energyaware workload assignment in data centers. The 2018 International Conference on High Performance Computing and Simulation, 8(5):811-817, 2018

[11] A. Liang, L. Xiao, Y. Pang, Y. Li, L. Ruan. Thermal-aware workload distribution for clusters. Procedia Engineering, 15:3308-3312, 2011

[12] P. Arroba, J. M. Moya, J. L. Ayala, R. Buyya. DVFS-aware consolidation for energy-efficient clouds. The 2015 International Conference on Parallel Architecture and Compilation, 494-495, 2016

[13] C. Q. Choi. "Reducing the massive energy appetite of data centers." Inside Science, URL https://www.insidescience.org/news/ reducing-massive-energy-appetite-data-centers. 22 Jan 2020.

[14] S. Xu, S. Lee, S.W Jun, M. Liu, J. Hicks, Arvind. BlueCache: A scalable distributed flash-based key-value store. Proceedings of the VLDB Endowment, 10(4):301-312, 2016

[15] D. Meisner, B. T. Gold, T. F. Wenisch. PowerNap: Eliminating server idle power. 44(3):205-216, 2009 
[16] Q. Tang, S. K. S. Gupta, G. Varsamopoulos. Thermal-aware task scheduling for data centers through minimizing heat recirculation. 2007 IEEE International Conference on Cluster Computing, 129-138, 2007

[17] E. Pakbaznia, M. Pedram. Minimizing data center cooling and server power costs. Proceedings of the 2009 ACM/IEEE International Symposium on Low Power Electronics and Design, 145-150, 2009

[18] A. Krioukov, P. Mohan, S. Alspaugh, L. Keys, D. Culler, R. Katz. NapSAC: Design and implementation of a power-proportional web cluster. Proceedings of the First ACM SIGCOMM Workshop on Green Networking, 15-22, 2010

[19] M. Lin, A. Wierman, L. L. H. Andrew, E. Thereska. Dynamic rightsizing for power-proportional data centers. IEEE/ACM Transactions on Networking (TON), 21(5):1378-1391, 2013

[20] D. Meisnert, C. M. Sadler, L. A. Barroso, W.D. Weber, T. F. Wenischt. Power management of online data-intensive services. ACM SIGARCH Computer Architecture News, 39(3):319-330, 2011

[21] "Load balancing (computing)." Wikipedia, URL https: //en.wikipedia.org/wiki/Load_balancing_(computing). 22 Jan 2020 .

[22] "Designing and managing a data center: Consolidation and virtualization.", Anixter, URL https://www. anixter.com/en_ca/resources/literature/techbriefs/ 
data-center-consolidation-and-virtualization.html. 22 Jan 2020.

[23] CLEAResult. "80 PLUS certified power supplies and manufacturers." Plug Load Solutions, 2019, URL https://www.plugloadsolutions. com/80PlusPowerSupplies.aspx. 22 Jan 2020.

[24] Hewlett-Packard. "HP common slot power supply technology." Technology Brief, 3rd edition, 2012

[25] "Strategy for carbon emissions reduction in inner city campuses", URL http://www.esru.strath.ac.uk/EandE/Web_sites/ 15-16/Low_Carbon_Campus/Group_C_IT_Equipment.html

[26] "Concave function." Wikipedia, URL https://en.wikipedia.org/ wiki/Concave_function. 22 Jan 2020. 


\section{A Appendix 1}

Here we are going to determine the workload assignment algorithm when $n=2$ which means $G(\bar{x})$ is a function of just one variable. By using (3), $G(\bar{x})$ is as follows:

$$
\begin{aligned}
G(\bar{x}) & =g\left(x_{1}\right)+g\left(L-x_{1}\right) \\
& =(3 A L+2 B) x^{2}-(3 A L+2 B) x+\left(A L^{3}+B L^{2}+C L\right)
\end{aligned}
$$

So $G(\bar{x})$ is a quadratic function. To minimize $G(\bar{x})$, we need to find the solution of $G^{\prime}\left(x_{1}\right)=0$.

$$
\begin{aligned}
G^{\prime}\left(x_{1}\right) & =-(L-2 x)(3 A L+2 B)=0 \\
x & =\frac{L}{2}
\end{aligned}
$$

Based on the second derivative of $G\left(x_{1}\right)$ we can determine if the function is an upward quadratic function. If it is, then $x=\frac{L}{2}$ is the minimum, otherwise it is the maximum.

$$
G^{\prime \prime}\left(x_{1}\right)=6 A L+4 B
$$

So if $L>-\frac{2 B}{3 A}=2 m$ then $G^{\prime \prime}\left(x_{1}\right)>0$ so $x=\frac{L}{2}$ is the minimum which means assigning the load $L$ equally to both servers minimizes $G(\bar{x})$. On the 
other hand, if $L<2 m$ then $x=\frac{L}{2}$ is the maximum of $G(\bar{x})$. In this case, we can use the properties of concave functions to find the minimum. In concave functions we have:

$$
g(a)+g(b) \geq g(a+b)
$$

So if we are able to assign the total load $L$ to one of the two servers and turn the other server off, that would be the minimum of $G\left(x_{1}\right)$ when $L<2 m$. Recall in Lemma 2, we assumed that $m<\frac{V}{2}$ so $2 m<V$, on the other hand, $L<2 m$ so $L<V$ which means we are able to assign the whole load to a server. So when $L<2 m$, by turning a server off and assigning $L$ to the other server, $G(\bar{x})$ is minimized. 